\title{
Blue and yellow long-period variables in the direction of the Small Magellanic Cloud ${ }^{\star}$
}

\author{
R. E. Mennickent ${ }^{1}$, L. Cidale $^{2}$, G. Pietrzyński ${ }^{1,3}$, W. Gieren ${ }^{1}$, and B. Sabogal ${ }^{1}$ \\ 1 Universidad de Concepción, Departamento de Física, Casilla 160-C, Concepción, Chile \\ e-mail: rmennick@astro-udec.cl, pietrzyn@hubble.cfm.udec.cl, wgieren@astro-udec.cl, bsabogal@udec.cl \\ 2 Facultad de Ciencias Astronómicas y Geofísicas, Univ. Nacional de La Plata, Paseo del Bosque S/N, 1900 CGA, La Plata, Argentina \\ e-mail: lydia@fcaglp.fcaglp.unlp.edu.ar \\ 3 Warsaw University Observatory, Al. Ujazdowskie 4,00-478, Warsaw, Poland
}

Received 4 April 2006 / Accepted 27 May 2006

ABSTRACT

\begin{abstract}
Aims. We investigate the nature of a sample of 17 long-term periodic variables in the direction of the Small Magellanic Cloud. Methods. Based on new spectroscopic data, we determined spectral types, radial velocities, absolute magnitudes, and colors for these stars. We present a refined discussion of their OGLE light curves along with an analysis of their 2MASS photometry.

Results. Most stars turned out to be B-A giants members of the Small Magellanic Cloud. We find a new interacting eclipsing binary with a period of 184 days and two new early-type ellipsoidal variables. One of our objects is the ROSAT source RX J0058.2-7231. We analyzed 11-years of data for this Be X-ray binary finding that their photometric period varies by $\sim 4 \%$ quasiperiodically, on a time scale of $\sim 1200$ days. We find evidence of multiple photometric periods in 2 Ae-type and 1 late-Be type stars. The case of OGLE00445466-7328029 is especially interesting, as this late-type Be star shows a beating phenomenon primarily caused by two closely-spaced frequencies, $0.05733 \mathrm{c} / \mathrm{d}(17.44 \mathrm{~d})$ and $0.06347 \mathrm{c} / \mathrm{d}(15.76 \mathrm{~d})$. Four other emission-line objects show stable longterm periodicities and probably correspond to Be-star binaries. Transient photometric periods are only found in 4 non-emission early-type stars.
\end{abstract}

Key words. stars: binaries: general - stars: binaries: eclipsing - stars: early type - stars: emission-line, Be - stars: circumstellar matter - stars: mass-loss

\section{Introduction}

From an inspection of the OGLE-II photometric database (Zebrun et al. 2001), while searching for Be stars in the Small Magellanic Cloud (SMC), Mennickent et al. (2002, hereafter M02) found 78 variable stars characterized by periodic photometric variability. As noted by these authors, the nature of these variables is still unclear. They are redder than typical Be stars, usually show variable amplitudes, and subsequent research has shown that their periods clearly show a bimodal distribution (Mennickent et al. 2003) with a main broad peak between 20 and 130 days and a narrower secondary peak between 140 and 210 days. The same kind of type-3 stars were found in the Large Magellanic Cloud, and they also show a bimodal period distribution (Sabogal et al. 2005). As noted by M02, the photometric variability is very different to that observed in any known pulsating variable in the same color range, like SPBs or $\alpha$ Cyg stars.

From these investigations several interesting questions arise, for instance: What kind of phenomenon is triggering the photometric activity in these stars? Why is a bimodal period distribution for type-3 variables observed in both galaxies? In order to contribute to answering these very fundamental questions we present here the first spectroscopic and detailed photometric study of a sub-sample of bright type-3 stars. We selected objects with $V$ magnitudes in the range 13.4-15.5 mag. In Sect. 2, we give an overview of the photometric and spectroscopic

* Figures 3, 5-7, 9-13, 15-17 and Tables 1 and 3 are only available in electronic form at http://www. edpsciences.org observations, along with a brief description of the methods employed in the analysis. In Sect. 3 we present the results of our study. We discuss our results in Sect. 4, and give conclusions in Sect. 5.

\section{Spectroscopic observations}

Spectroscopic observations were conducted at CTIO during October 8-12, 2003 (UT). We used the $1.5 \mathrm{~m}$ telescope with the Cassegrain Spectrograph and the Loral $1 \mathrm{~K}$ detector. The first two nights, the grating \#26 tilted at 16.2 degree and a slit width of 2 arcsecond yielded a spectral range of 3700-5470 , a dispersion of $1.3 \mathrm{arcsec} / \mathrm{pix}$, and an effective resolution of $2 \AA$. The Loral CCD had a few dead pixels around wavelength $4051 \AA$; the artifacts produced by these pixels in the spectra were removed by interpolating the flux of nearby pixels. During the third and fourth nights, we used grating \#36, with the same slit width, which yielded a dispersion of $0.77 \AA /$ pix, a $1.9 \AA$ effective spectral resolution, and a wavelength range of 5765-6694 A. For this setup, the CCD dead zone was between 5948 and $5951 \AA$. As for the blue spectra, bad pixels were removed by interpolating the flux between nearby pixels. The He-Ar comparison spectra were obtained between several science exposures typically every half hour. These comparison spectra provided wavelength calibration functions with typical rms of $0.1 \AA$ for grating \#26, and $0.01 \AA$ for grating \#36 $\left(6 \mathrm{~km} \mathrm{~s}^{-1}\right.$ and $0.5 \mathrm{~km} \mathrm{~s}^{-1}$ at $\mathrm{H} \beta$ and $\mathrm{H} \alpha$, respectively). Observations of the standard star LTT 2415 were 
Table 2. The $V$ magnitude provided by the OGLE catalogue is given, along with our determinations for spectral type, luminosity class, absolute magnitude, color and radial velocity.

\begin{tabular}{|c|c|c|c|c|c|c|c|c|}
\hline OGLE-name & ID & $V$ & $(B-V)_{0}$ & $M_{V}$ & $v \pm \sigma_{V}$ & $N$ & ST & Note \\
\hline OGLE00433691-7326377 & SMC3-1 & 14.18 & & & $114 \pm 31$ & 15 & A7-F5e & $M$ \\
\hline OGLE00445466-7328029 & SMC3-2 & 14.82 & & & $137 \pm 26$ & 7 & B7-9 IIIe & $M$ \\
\hline OGLE00455414-7314043 & SMC3-3 & 15.48 & & -2.66 & $174 \pm 23$ & 7 & F0 Ib-II & $S$ \\
\hline OGLE00463376-7312043 & SMC3-4 & 14.06 & & & $126 \pm 15$ & 1 & $\mathrm{~F} 5 \mathrm{Ie}+\mathrm{G} 0 \mathrm{I}$ & $E 1$ \\
\hline OGLE00475014-7313164 & SMC3-5 & 15.48 & & -2.05 & $139 \pm 5$ & 7 & B7-8 III & $T$ \\
\hline OGLE00492141-7258449 & SMC3-6 & 14.61 & & -0.84 & $191 \pm 23$ & 13 & A5 II & $T$ \\
\hline OGLE00502564-7258071 & SMC3-7 & 14.01 & & & $127 \pm 15$ & 10 & F4 IV & $T$ \\
\hline OGLE00504344-7327053 & SMC3-8 & 13.65 & & & $179 \pm 26$ & 4 & WR & Wolf-Rayet \\
\hline OGLE00510018-7253039 & SMC3-9 & 13.61 & & & $172 \pm 32$ & 12 & B1 II-IIIe & $S$ \\
\hline OGLE00510759-7326366 & SMC3-10 & 15.05 & & & $138 \pm 18$ & 1 & & $E 2$ \\
\hline OGLE00535922-7235089 & SMC3-12 & 14.40 & & -0.01 & $54 \pm 17$ & 12 & A3 III & $E 2$ \\
\hline OGLE00552027-7237101 & SMC3-14 & 14.88 & & & $141 \pm 16$ & 2 & A0 Ibe & $M$ \\
\hline OGLE00574525-7235321 & SMC3-15 & 13.82 & -0.23 & -4.33 & $195 \pm 37$ & 22 & B1 II-III & $T$ \\
\hline OGLE00581258-7230485 & SMC3-16 & 14.90 & & & $164 \pm 24$ & 4 & B0 IIIe & Be X-ray \\
\hline OGLE01000078-7255229 & SMC3-18 & 14.44 & & & $222 \pm 32$ & 14 & B8-9 IIIe & $S$ \\
\hline OGLE01045121-7246469 & SMC3-20 & 13.42 & -0.21 & -4.32 & $179 \pm 32$ & 16 & B1 Ib-IIe & $S$ \\
\hline OGLE01045299-7159188 & SMC3-21 & 14.22 & -0.18 & -4.36 & $143 \pm 24$ & 15 & B2 IIIe & $S$ \\
\hline
\end{tabular}

used to calibrate the spectra in absolute flux units. The rms of the sensitivity function was 0.05 mag for grating \#26 and $0.02 \mathrm{mag}$ for grating \#36. Corrections for atmospheric extinction were done for every spectrum using average extinction coefficients available for CTIO. Spectroscopic reductions were carried out in the usual manner, using several IRAF routines like $c c d p r o c$ and doslit. A summary of the spectroscopic observations is given in Table 1.

\section{Methods and results}

In this section we present the methods used for spectral classification, give details on our search for photometric periodicities, provide a brief discussion of every object and discuss the available infrared photometric data.

\subsection{Spectral classification and radial velocities}

We measured radial velocities and equivalent widths of several lines in the spectra. The spectral classification of each star was derived by comparing it with the MK standard classification system, using the digital spectra available online (http://stellar.phys.appstate.edu/Standards/ stdCTIO.html). In the case of early-type stars, we also determined the spectral classification by using the measurements of the equivalent widths of the strongest lines (Didelon 1982). We mainly used lines of He I, Si II, Si III, Fe II, and Mg II. In a few cases we used the absorption lines of $\mathrm{H} \alpha$ and $\mathrm{H} \beta$ to confirm the classification, but in general we avoided them since they were usually filled by emissions. For many stars we were able to estimate absolute magnitudes $\left(M_{V}\right)$ and intrinsic colors, $(B-V)_{0}$. For B-type stars we adopted the equivalent widths of some selected pairs of He I lines (Stock et al. 2002) and for A, $\mathrm{F}$, and G-types we chose the metallic lines and the $\mathrm{H} \delta$ line (Stock \& Stock 1999). We could not determine fundamental parameters for some stars since the lower members of $\mathrm{H}$ lines displayed emission features. Our results are listed in Table 2. There $N$ is the number of lines averaged for the radial velocity determination and a note about photometric variability is provided: $M$ stands for multiperiodic, $T$ for transient periodicity, $E 1$ for eclipsing binary, $E 2$ for ellipsoidal variable and $S$ for single period variable.
We observe that the determinations of $M_{V}$ and $(B-V)_{0}$ agree with the spectral types that were derived independently. The major uncertainties may be present in late-B, A, and early-F types due to the presence of weak metallic lines and emissions in $\mathrm{H} \delta$ and $\mathrm{H} \gamma$. We note that the five early B-type stars show similar continuum slopes (see Sect. 3.3); a ratio $F_{\lambda 3500} / F_{\lambda 5500}$ between 2 and 2.7, except the enigmatic star SMC3-9, that shows a remarkable down turn in flux downwards of about $4500 \AA$. We observe this fact as a confirmation of the similar spectral type and the presence of reddening. As a reference, black-bodies with temperatures of 20000,13000 , and $10000 \mathrm{~K}$ have the aforementioned ratios equal to $3.8,2.7$, and 2 , respectively.

\subsection{Search for periodicities}

Our sample consists of stars whose photometric periods were first determined by M02. As that was a seminal paper, focused on the discovery of new types of variables in the Small Magellanic Cloud, no further detailed information was given about the possible multiperiodicity of the stars. In this section we describe our method of searching for multiple frequencies in the OGLE time series and list the results. The photometric data discussed here is part of the OGLE (Zebrun et al. 2001) database, and it can be accessed at http://www. astrouw.edu.pl/ ogle. The OGLE dataset consists of $I$-band time series observed between HJD 2450466 and HJD 2451757 (3.5 years), typically with 271-306 observations per star. To test the stability of our solutions, we also checked if the frequencies we found were also present in the OGLE-III database (Pietrzyński, private communication). This database now consists of four additional years of observations, which have almost been reduced at the moment of the writing of this paper, but lacking reliable zero points.

During our investigation of frequencies in the time domain, we used a computer program especially dedicated to the statistical analysis of large astronomical time series containing time gaps, the Period04 program first developed by Martin Sperl (Lenz \& Bregel 2005, http://www . astro. univie.ac . at/ dsn/dsn/Period04/).

First, we got the stronger frequency in the signal by applying a fast Fourier transform to the data. The analysis was done between $0 \mathrm{c} / \mathrm{d}$ and the Nyquist threshold. The main peak of the periodogram, i.e. those with the largest amplitude, was selected 
as our first frequency, labeled F1. A least-square sinus fit was then performed on the data with the function:

$Z+\sum A_{i} \sin \left(2 \pi\left(\Omega_{i} t+\Phi_{i}\right)\right)$

where $Z$ is the photometric average, $t$ the time, and $\Omega_{i}, A_{i}$, and $\Phi_{i}$ are the frequency, amplitude, and phase associated to the component $i$ of the least-square solution. For the first iteration we fixed $\Omega_{1}=\mathrm{F} 1$ and determined $A_{1}$ and $\Phi_{1}$ with the least-square fit routine. In a second step, the program also offers the possibility of improving all parameters, giving a better value for the frequency F1. We calculated the standard deviation of the fit $(\sigma)$ and obtained the residuals. These residuals were analyzed as a new dataset applying the fast Fourier transform. A new frequency $\mathrm{F} 2$ was obtained, then a new fit with $i=1$ and 2 was found for the original time series, and we got a new set of improved frequencies, amplitudes, and phases. Then we recalculated residuals with the new fitting function and continued this process successively until the last significant frequency was found. We considered a peak in the periodogram significant if it had an amplitude larger than four times the noise level (Breger et al. 1993; Aerts \& Kolenberg 2005). As noted by these authors, this criterion is more conservative than using power (amplitude squared) rather than amplitudes. The noise level was measured by averaging the peak amplitudes of the periodogram constructed after prewhitening the data with all significant frequencies. In general, this method was fairly successful, although, in some cases, successive prewhitening with significant frequencies did not improved the fit much and gave unreliable lowamplitude frequencies. For this reason, we rejected frequencies whose amplitudes were smaller than $0.003 \mathrm{mag}$, and also used one additional criterion to establish the validity of the remaining significant frequencies; they should also be present in the OGLE-III dataset, i.e., during the four additional years of observations. This "stability test" was quite useful for cleaning the frequencies that passed the test of significance. Frequencies that do not pass this criterion could be artifacts of the prewhitening process, or they could indicate short-lived periodic phenomena.

We calculated uncertainties in the fit parameters using Monte Carlo simulations. We constructed 100 trials of synthetic datasets, determined solutions for every one, and calculated the standard deviation of frequency, amplitude, and phase. The results of our analysis are summarized in Table 3, which gives the frequencies, amplitudes ( $A$, in mmag), and phases, along with their uncertainties. The table also gives the noise level for every star and the standard deviation of the fit $\sigma$ for every new frequency added, and a flag indicating whether the frequency passed the stability test.

In some cases, we obtained unrealistically high errors associated to the amplitudes of the sinusoidal solutions. We interpreted these results as unstable solutions, which could be due to formal, rather than physical, reasons. For these cases, we calculated uncertainties from the error matrix of the least-square calculation.

\subsection{Comments on individual stars}

We cross-correlated our targets with stellar catalogues using the SIMBAD on-line query form. A number of matches were found that correspond to 7 previously catalogued emissionline objects (from $\mathrm{H} \alpha$ imaging surveys), one Wolf Rayet star (OGLE00504344-7327053 = AzV 81), one Be X-ray binary (OGLE00581258-7230485 = RX J0058.2-7231), and the nonemission object SMC41243 (Massey 2002). A brief description of the results for individual objects and a summary of the literature search is given below.

\subsubsection{OGLE00433691-7326377 (SMC3-1)}

This member of the SMC open cluster NGC 242 was previously known as the $\mathrm{H} \alpha$ emission object LHA $115-\mathrm{S} 3$ (Henize 1956). It was classified "type-3" by M02 due to the periodic pattern in the OGLE light curve with a periodicity of $118 \pm 7$ days (Fig. 1a). The spectrum shows double-peaked $\mathrm{H} \alpha$ emission with equivalent width $-99 \AA$ and the red peak higher than the violet one $(V<R)$. We also observed $\mathrm{H} \beta$ emission with equivalent width $-8.3 \AA$, higher-order Balmer lines filled by emission, and many metallic absorption lines. All this evidence supports the existence of an optically thin gaseous envelope around the star. From the blue spectral region, we derived a spectral type between A7 and F0. The red spectrum is similar to those of SMC3-4 (classified F5 I, see below). We conclude that the spectral type is between A7 and F5 but that the absolute magnitude and luminosity are uncertain. A Gaussian fit to the $\mathrm{NaD}$ lines observed in the red spectrum gives equivalent widths of $\mathrm{NaD} 1=1.25 \AA$ and $\mathrm{NaD} 2=0.86 \AA$, i.e., a ratio marginally closer to 1.1 . This could suggest that the star is embedded in gas of high optical depth (e.g. Munari \& Zwitter 1997). At our resolution, the Na D lines appear as single components, so we could apply the calibration between their equivalent width and the reddening found by Munari \& Zwitter (1997). However, the extremely large equivalent width should imply $E(B-V)>1$, yielding an very unrealistic blue color. We conclude that the $\mathrm{Na} \mathrm{D}$ line likely include multiple, not resolved, lines of probably interstellar origin.

Our method of searching periodicities yielded 7 significant frequencies (Fig. 1b); the most significant are $0.00844 \pm$ $0.00044 \mathrm{c} / \mathrm{d}, 0.06498 \pm 0.00002 \mathrm{c} / \mathrm{d}$, and $0.01261 \pm 0.00005 \mathrm{c} / \mathrm{d}$. The other four frequencies, $0.02115 \pm 0.00007 \mathrm{c} / \mathrm{d}, 0.00416 \pm$ $0.00204,0.02932 \pm 0.00975$, and $0.05439 \pm 0.00565 \mathrm{c} / \mathrm{d}$, improved the fit only marginally. We first suspected that only the existence of the first three frequencies was well supported; F2 improved $\sigma$ by $26 \%$ and $\mathrm{F} 3$ by $14 \%$. However, all frequencies, except F7, are also present in the OGLE-III dataset, confirming the presence of 6 periods in this variable star.

The $\mathrm{H} \alpha$ emission with equivalent width of around $-100 \AA$ could suggest a Herbig Ae/Be object. The pre-main sequence classification is in principle consistent with the membership of this star in the open cluster NGC 242 and the relatively young cluster age, viz. $20 \pm 10 \mathrm{Myr}$ (Ahumada et al. 2002). We collected $J, H, K$ magnitudes from the 2MASS survey and found $J-H=0.20 \pm 0.06$ and $H-K=0.07 \pm 0.06$. Although these figures could indicate a small or null infrared excess (a galactic F0 supergiant has $J-H=0.15$ and $H-K=0.03$, Koornneef 1983), this is not enough evidence to reject the Herbig Ae/Be classification in the low metallicity environment of the $S M C$ (de Wit et al. 2003, 2005). However, we note that the amplitude of variability ( $\sim 0.02 \mathrm{mag}$ ) is small for Herbig Ae/Be objects, which typically show irregular variability with amplitude of $\sim 0.1 \mathrm{mag}$.

\subsubsection{OGLE00445466-7328029 (SMC3-2)}

No previous study was found for this object. The OGLE data indicate a periodic star with a light curve of modulated amplitude, produced primarily by the beating of two closely-spaced frequencies, $0.05733 \mathrm{c} / \mathrm{d}(17.44 \mathrm{~d})$ and $0.06347 \mathrm{c} / \mathrm{d}(15.76 \mathrm{~d})$ (Fig. 2a, M02). No other star in our sample shows such a 
HJD - 2,400,000
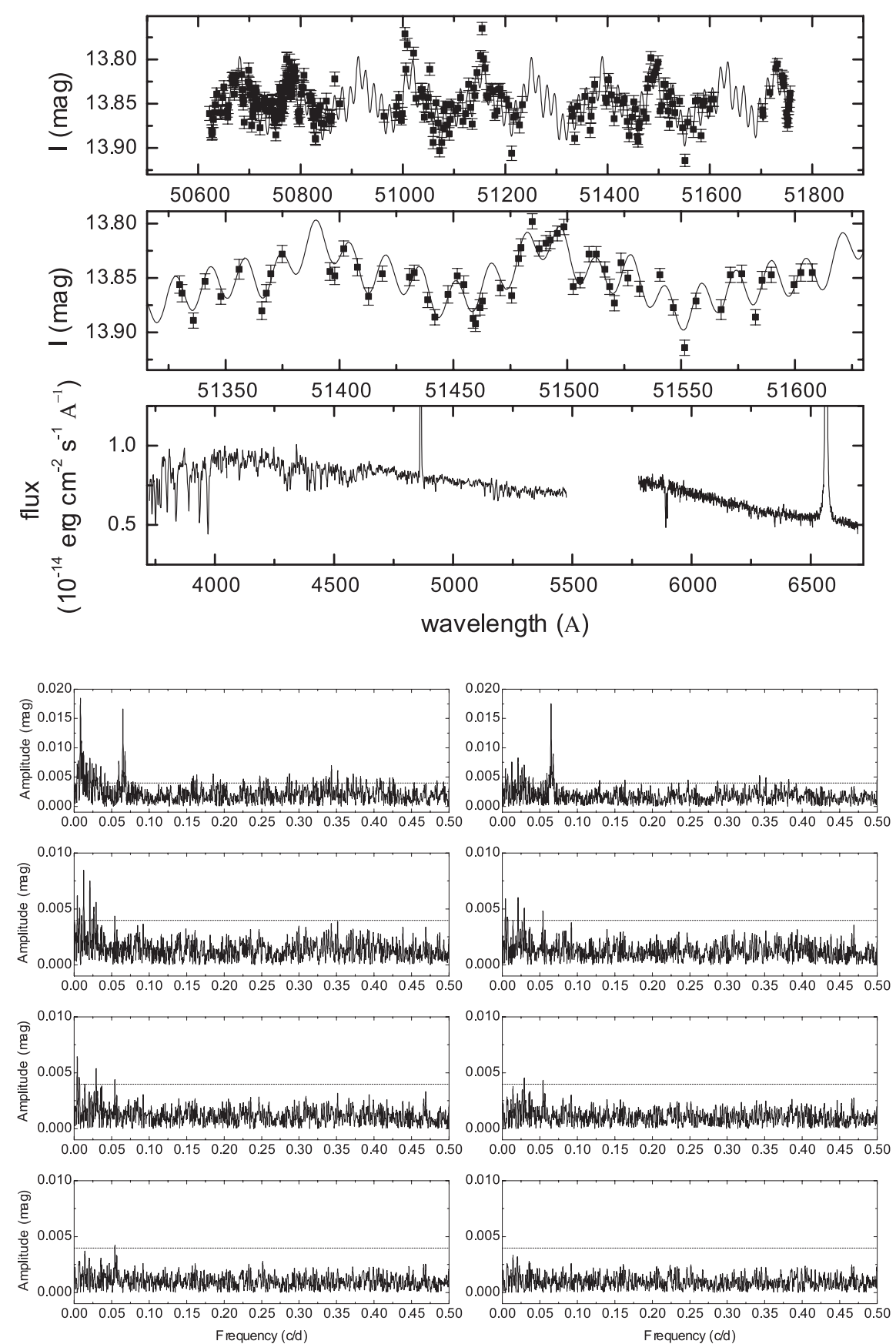

Fig. 1. a) OGLE light curve and the best-fitting function (upper), detail of the light curve and fit (middle) and the spectrum of SMC3-1 (bottom). b) Fourier spectrograms illustrating the prewhitening process for SMC3-1. The dotted line indicates the significance level determined as explained in the text. This figure and subsequent ones should be read from up to down and from left to right.

remarkable phenomenon. The spectrum shows $\mathrm{H} \alpha$ emission with an equivalent width of $-6.5 \AA$ and other Balmer lines filled by emission. We found a spectral type of B 7-9 III e, but the spectrum lacked the signatures to determine the color and absolute magnitude. The infrared colors for this star compiled from the 2MASS catalogue are $J-H=0.32 \pm 0.09$ and $H-K=0.11 \pm$ 0.09 , which when compared with typical colors for a (galactic) B9 III star, namely $J-H=-0.02$ and $H-K=0.02$ (Winkler 1997), indicate significant reddening at the $J-H$ color.

Our frequency-searching method yielded 5 frequencies for this star below the Nyquist threshold of $0.92 \mathrm{c} / \mathrm{d}$. These are listed in Table 3. Although all these frequencies satisfy our criterion having amplitudes more than 4 times the noise level, only F1, $\mathrm{F} 2$, and F3 produce a rapid and significant improvement in the fit quality (as measured by $\sigma$ ). The calculated fit reproduces the beating observed in the light curve very well (Fig. 2a). In spite of the fact that F4 and F5 improve the fit only marginally, all frequencies are confirmed by OGLE-III data, which confirms the multiperiodicity of this star.

We note the similarity between the light curve of SMC3-2 and those of RX UMa, a galactic RV-Tau type semiregular variable (Kiss et al. 2000). The variability of these stars differs 

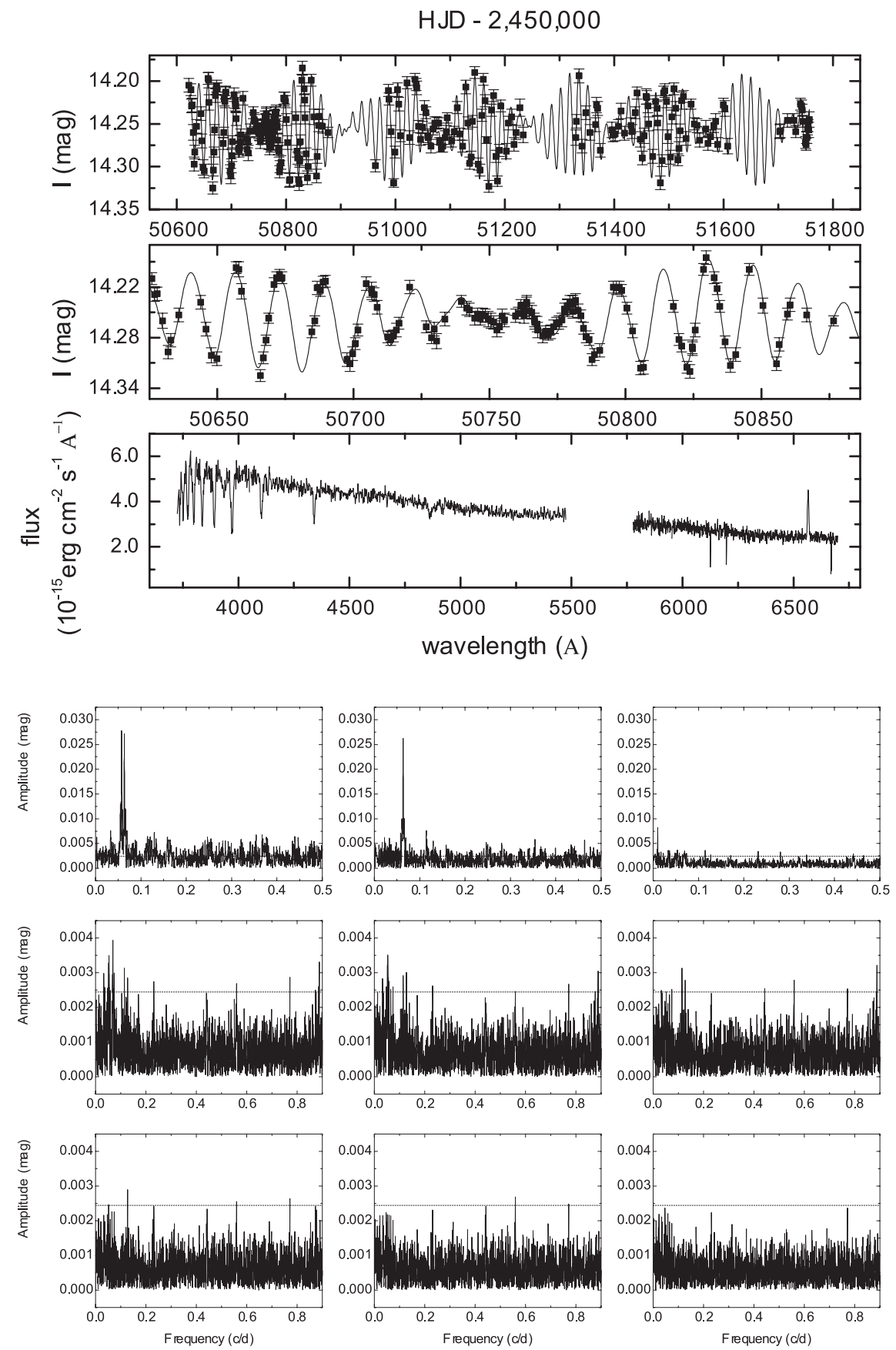

Fig. 2. a) OGLE light curve and the best-fitting function (upper), detail of the light curve and fit (middle) and spectrum (bottom) of SMC3-2. b) Fourier spectrograms illustrating the prewhitening process for SMC3-2. The dotted line indicates the significance level determined as explained in the text.

only by a scale factor, the variability of RX UMa having larger amplitude and longer time scale than that of SMC3-2. Kiss et al. (2000) explain the behavior of RXUMa as a beating of two closely-separated modes of pulsation. According to these authors, the period ratio (close to the ratio 1.107 found in SMC3-2) suggests either high-order overtone or radial+nonradial oscillation. Contrary to SMC3-2, however, semiregular variables are usually highly unstable in coherence and amplitude of variability and have cooler spectral types.

We see three possible interpretations for the beating phenomenon: (i) it could be caused by modulated light variations in a three-body system; (ii) it could be caused by two protoplanets surrounded by dust shells orbiting at nearby radii as measured from the central star; and (iii) it could reflect some kind of stellar pulsation. Alternative (i) is unlikely since the volume of the putative B-type binary probably excludes another possible binary with a period on the order of 15 days. Alternative (ii) is attractive and predicts larger amplitudes in redder passbands. This is what we really observe. The analysis of the MACHO instrumental $b$ and $r$ photometry indicates that the $b-r$ color oscillates with the same two main frequencies as the $I$-band magnitude, but with amplitudes of $0.007 \pm 0.001 \mathrm{mag}(\mathrm{F} 1)$ and $0.010 \pm$ $0.001 \mathrm{mag}(\mathrm{F} 2)$, and the star is redder when brightest.

The problem with this view is that two protoplanets orbiting at close orbits would be gravitationally unstable, hence the probability of finding such a system is quite low. Finally, 

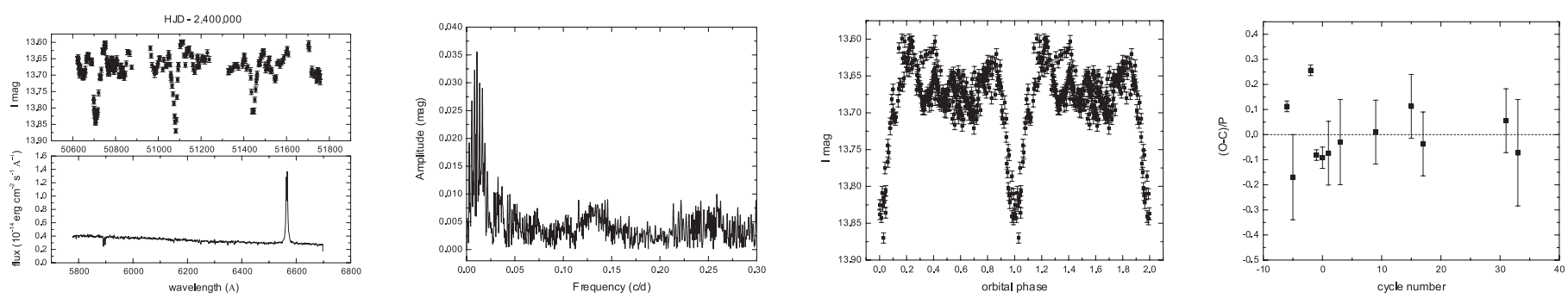

Fig. 4. a) OGLE light curve (upper) and spectrum (bottom) of SMC3-4. b) Fourier spectrogram for SMC3-4. c) Light curve folded with the period of 184 days. d) $\mathrm{O}-\mathrm{C}$ diagram for local minima outside eclipse; the test period is 23.5658 days.

alternative (iii) is hard to admit, since there is no pulsation mechanism known in B-type stars acting with such a time scale of variability (Pamyatnykh 1999). If some kind of unknown pulsation is assumed, along with binarity, then the modulation could reflect the misalignment between the orbital axis and the pulsational axis, produced by the tidal force of the faint stellar component. In fact, Reed \& Brondel (2005) report modulated light curves in these cases, very similar to that shown in Fig. 2. Although their analysis was performed for close binary systems with short orbital periods, we can scale their results to our system. Their results show that for some inclinations (see their Fig. 1) two closely spaced frequencies are obtained as the orbital aliases of the original pulsational frequency. In our case, the pulsational frequency should be $(0.05733+0.06347) / 2 \mathrm{c} / \mathrm{d}$, i.e. $0.0604 \mathrm{c} / \mathrm{d}$ (16.556 days), and the orbital frequency $0.00307 \mathrm{c} / \mathrm{d}$, i.e. 325.73 days. In this scenario, the doubt about the origin of the pulsation still remains, as does the question of which component could tilt the pulsational axis of the B-type star in spite of the large orbital separation. It is also possible that the pulsation axis is tilted with respect to the rotational axis without invoking the assumption of a binary companion, as in rapidly oscillating Ap (roAp) stars (Baldry et al. 1998). However, this case should produce frequency triplets, not doublets.

We note that the red spectrum of SMC3-2 shows peculiar absorption lines at $\lambda \lambda$ 6125.6, 6197.1, 6300.1, 6319.2, and $6431.5 \AA$, which we have tentatively identified (considering a stellar velocity of $137 \mathrm{~km} \mathrm{~s}^{-1}$ ) as Ca I 6122.2 (it could also be Mn II 6122.4, 6122.8 and 6123.2), Zr III 6193.7 (or Sc I 6193.7), Fe I 6297.8 (or Ti I 6296.6, 6298.0), Ni I 6316.6 (or Fe I 6315.8), and Fe I 6428.8. These lines could be formed in the gaseous envelope, but they could also indicate enhanced chemical abundances as in magnetic Bp stars. Further high-resolution spectroscopy is needed to test the possible magnetic nature of this variable.

\subsubsection{OGLE00455414-7314043 (SMC3-3)}

The OGLE light curve shows a noisy periodic pattern (Fig. 3a), and M02 report a low amplitude periodicity of $99 \pm 4$ days. No emission is detected in our spectrum, which indicates a spectral type of F0 Ib-II. In agreement with M02, we found only one significant frequency, $\mathrm{F} 1=0.00983 \pm 0.00005$, which is also present in the OGLE-III database. Fourier spectrograms are given in Fig. 3b.

\subsubsection{OGLE00463376-7312043 (SMC3-4)}

This variable was previously classified as the emission line object Lin-81 (Lindsay 1961). The OGLE light curve reveals an unusual eclipsing binary with a period of $184 \pm 18$ days (Figs. 4a,b, M02). The depth of the minima and the general appearance of the light curve are quite variable. The presence of additional oscillations outside the principal eclipse makes it difficult to identify the secondary eclipse. However, an inspection of the phase diagram, constructed with the ephemeris $P=184$ days and $T_{\min }=50523.7769(\mathrm{MJD})$, suggests that the secondary minimum, if present, should be $>0.18$ mag shallower than the main minimum. This indicates that the fainter component should have at most $83 \%$ of the temperature of the brighter component. The phase curve also shows that the oscillations outside the main minimum are not modulated with the binary period (Fig. 4c). Figure 4d shows the $\mathrm{O}-\mathrm{C}$ diagram (observed minus calculated, see Sterken 2005) for these local minima, with a test period of 23.5658 days; some outliers in the graph suggest quasiperiodicity for this short-term variability.

The $\mathrm{H} \alpha$ emission is double-peaked, with $V \approx R$, and an equivalent width of $-31 \AA$. Their wings extend to $-760 \mathrm{~km} \mathrm{~s}^{-1}$ and $700 \mathrm{~km} \mathrm{~s}^{-1}$ from the line centre. The double $\mathrm{H} \alpha$ emission is more consistent with emission from a rotationally supported, disc-like envelope, than from a stellar wind. As we do not have blue spectra for this star, we could not use the method described in Sect. 3.1 to find the spectral type. As an alternative method, we compared the red spectrum with the library of stellar spectra of Jacoby et al. (1984), excluding the regions around the $\mathrm{H} \alpha$ and the NaD lines. This yielded a spectral type of F5 I. A double Gaussian fit to the sodium D lines gives equivalent widths of $\mathrm{NaD} 1=1.15 \AA$ and $\mathrm{NaD} 2=0.93 \AA$, i.e., a ratio close to 1.1. This could suggest that the star is embedded in gas of high optical depth (Munari \& Zwitter 1997). We do not know if these lines are single or multiple, so we cannot use the relationship between equivalent width of single $\mathrm{NaD}$ lines and reddening given by these authors. If the lines were single, then their equivalent widths would indicate a very large reddening, larger than $E(B-V)=1$, but this yields an unrealistically blue color for the star. This suggest that the lines are formed at different regions and are multiple. This is consistent with the fact that the equivalent width of the $\mathrm{NaD}$ lines is larger than expected for a normal F5 I star. A contribution could come from the envelope responsible for the $\mathrm{H} \alpha$ emission. However, the velocity of the $\mathrm{Na}$ lines, viz. $79 \mathrm{~km} \mathrm{~s}^{-1}$, is different from those of $\mathrm{H} \alpha\left(126 \mathrm{~km} \mathrm{~s}^{-1}\right)$, suggesting the presence of another medium, maybe interstellar, or the companion star (see below), in the formation of the sodium D doublet.

That the eclipses are of variable depth and shape (Fig. 4c), and the strong variability of the light curve together suggest that the system could be an interacting spectroscopic binary. If we assume that the F5 supergiant is surrounded by an accretion disc fed by mass transferred from a secondary star filling its Roche-lobe, then the orbital period constrains the mean density of the mass donor star. Using standard formulae for close binary systems (e.g. Frank et al. 2002), we obtain $5.8 \times 10^{-6} \mathrm{~g} \mathrm{~cm}^{-3}$ 
for the mean density of the secondary star, which corresponds to a G0 supergiant.

\subsubsection{OGLE00475014-7313164 (SMC3-5)}

M02 report a low-amplitude periodicity of $30.0 \pm 0.3$ days (Fig. 5a). No emission was detected in our spectrum, which indicates a spectral type of B7-8 III. We found two frequencies, $0.03321 \pm 0.00003$ and $0.00135 \pm 0.00322$. F2 accounts for the long-term variability, while their large error and low amplitude, close to the noise level (Fig. 5b), suggest that F1 is the only real frequency in this star. However, neither F1 nor F2 are present in OGLE-III data. This suggests that we have detected a transient periodicity in this star.

\subsubsection{OGLE00492141-7258449 (SMC3-6)}

The OGLE light curve shows long-term periodic variability (Fig. 6a). M02 report a period of $197 \pm 20$ days. No emission is detected in our spectrum, which indicates a spectral type of A5 II. In agreement with M02, we found only one frequency, $0.00509 \pm 0.00155 \mathrm{c} / \mathrm{d}$ (Fig. 6b). However, this frequency is not reproduced by OGLE-III data, so we conclude that this star probably shows a transient periodicity.

\subsubsection{OGLE00502564-7258071 (SMC3-7)}

The OGLE light curve shows a noisy light curve with a rapid low-amplitude periodic oscillation (Fig. 7a). M02 gives a periodicity of $27.32 \pm 0.40$ days, which agrees with our finding of only one frequency, $0.03661 \pm 0.00105 \mathrm{c} / \mathrm{d}$ (Fig. $7 \mathrm{~b}$ ), but this is not reproduced by OGLE-III data, so it could be a transient periodicity. No emission is observed in our spectrum, which indicates a spectral type of F4 IV, locating this star in the Milky Way halo.

\subsubsection{OGLE00504344-7327053 (SMC3-8)}

This object was previously classified as the Wolf Rayet star AzV 81 (Massey 2002), and it corresponds to the star WR 4 in the list of Massey \& Duffey (2001), who classify it as WN6. From RV studies, the star seems to be single, rather than a binary (Foellmi et al. 2003; Moffat 1988). Its wind properties were studied by Crowther (2000) from optical and infrared spectra. The OGLE light curve shows a periodic variability of variable amplitude. Our spectrum shows typical emission lines of WR stars, especially the strong He II $\lambda 4686$ line (Fig. 8a). A list of emission line strength for this star is given in Table 4. For this object we found 6 significant frequencies, which are listed in Table 3. M02 report a periodicity of $62 \pm 2$ days, but a re-analysis of their data with the IRAF $p d m$ routine gives 51.8 days, in agreement with our first frequency. The difference probably corresponds to a typo in the M02 table. Even with these frequencies, the fit could not account for the variability in several epochs (Fig. 8b); this suggests the presence of aperiodic components in the signal.

Foellmi et al. (2003) reported a periodicity of 6.55 days in the blue MACHO data for this star, and no periodicity in the OGLE light curve. We have no explanation for why they missed the 52-day periodicity and the other associated frequencies (we do not find the 6.55-day period in our analysis). It is then possible that the wrong light-curve was analyzed by these authors. Unfortunately, we had no additional data from OGLE-III to track the long-term behaviour of this star. We investigated the possibility that the 52-day periodicity could be the binary period. In this case it would be the longest period among WR stars in the SMC (Foellmi et al. 2003). Consequently, the radial-velocity amplitude could be low and eventually undetectable. However, using masses of 10 and $30 M_{\odot}$ for the WR and O-type component, and an eccentricity of zero, the radial velocity half amplitude should still be detectable, between $60-150 \mathrm{~km} \mathrm{~s}^{-1}$ for inclination angles of 30-90 degres.

\subsubsection{OGLE00510018-7253039 (SMC3-9)}

This object was classified as the $\mathrm{H} \alpha$ emission object LHA 115-N 41 (Henize 1956) and is also named AzV 85 (Azzopardi \& Vigneau 1982). The OGLE light curve shows irregular variability that consists of modulated oscillations prior to HJD 2451600 and a sudden drop in brightness after this epoch (Fig. 9a). M02 report a period of $63 \pm 3$ days. We found Hydrogen and Helium lines in emission and determined a spectral type of B1 II-IIIe. The $\mathrm{H} \beta$ equivalent width is $-2.2 \AA$ (Fig. 9a), which is typical of Be stars. Fading events as the observed in SMC3-9 have also been reported in some galactic Be stars. For instance, the Be star $28 \mathrm{CMa}$ showed, during March/April 1988, a sudden fading of a few weeks duration that was preceded by a quasi-periodic oscillation in time scales of 25 days (Mennickent et al. 1994). Our analysis of the data prior to HJD 2451600 indicates the presence of three frequencies, $\mathrm{F} 1=0.00199 \pm 0.000272 \mathrm{c} / \mathrm{d}$, which removes the longterm tendency, $\mathrm{F} 2=0.01594 \pm 0.00288 \mathrm{c} / \mathrm{d}$, and F3 $=0.01743 \pm$ $0.00006 \mathrm{c} / \mathrm{d}$ (Fig. 9b). Only F2 is recurrent in the OGLE-III time series, and it is also the frequency reported by M02.

\subsubsection{OGLE00510759-7326366 (SMC3-10)}

The OGLE light curve reveals the periodic variability, which M02 reported as of low amplitude with a period of $185 \pm$ 17 days. Our spectra cover only the red range, $\mathrm{H} \alpha$ is seen as a weak absorption, and the continuum slope is rather blue (Fig. 10a). We detected only the frequency reported by M02, viz. $0.00541 \pm 0.00018 \mathrm{c} / \mathrm{d}$ (Fig. 10b), which is corroborated by OGLE-III data. However, the star could be an ellipsoidal variable if the true periodicity were twice F1. Based on the appearance of the phase diagrams, we favor this solution; we find an ephemeris for the times of a main minimum of $P=369.69$ days and $T_{\min }=$ 50303.3773 (MJD) (Fig. 10a).

\subsubsection{OGLE00535922-7235089 (SMC3-12)}

The OGLE light curve shows oscillations with variable amplitudes (Fig. 11a). M02 derive a period of $73 \pm 3$ days. No emission is observed in our spectrum. We estimate the spectral type A3 III (Fig. 11a). The low radial velocities could indicate that the object is a possible member of the galactic halo, but they could also be due to orbital motion in a binary star. Our analysis gives two frequencies satisfying the significance criterion, F1 = $0.01378 \pm 0.00002$ and $\mathrm{F} 2=0.00688 \pm 0.00006$ (i.e. $0.5 \mathrm{~F} 1$, Table 3). Both frequencies are confirmed by OGLE-III data. The solution with minima of alternating depth is compatible with a binary showing ellipsoidal variations with orbital frequency $0.00688 \mathrm{c} / \mathrm{d}$ (145.349 days) (Fig. 11a). We find an ephemeris for times of a main minimum of $T_{\min }=50330.26981$ (MJD). 
HJD $-2,400,000$
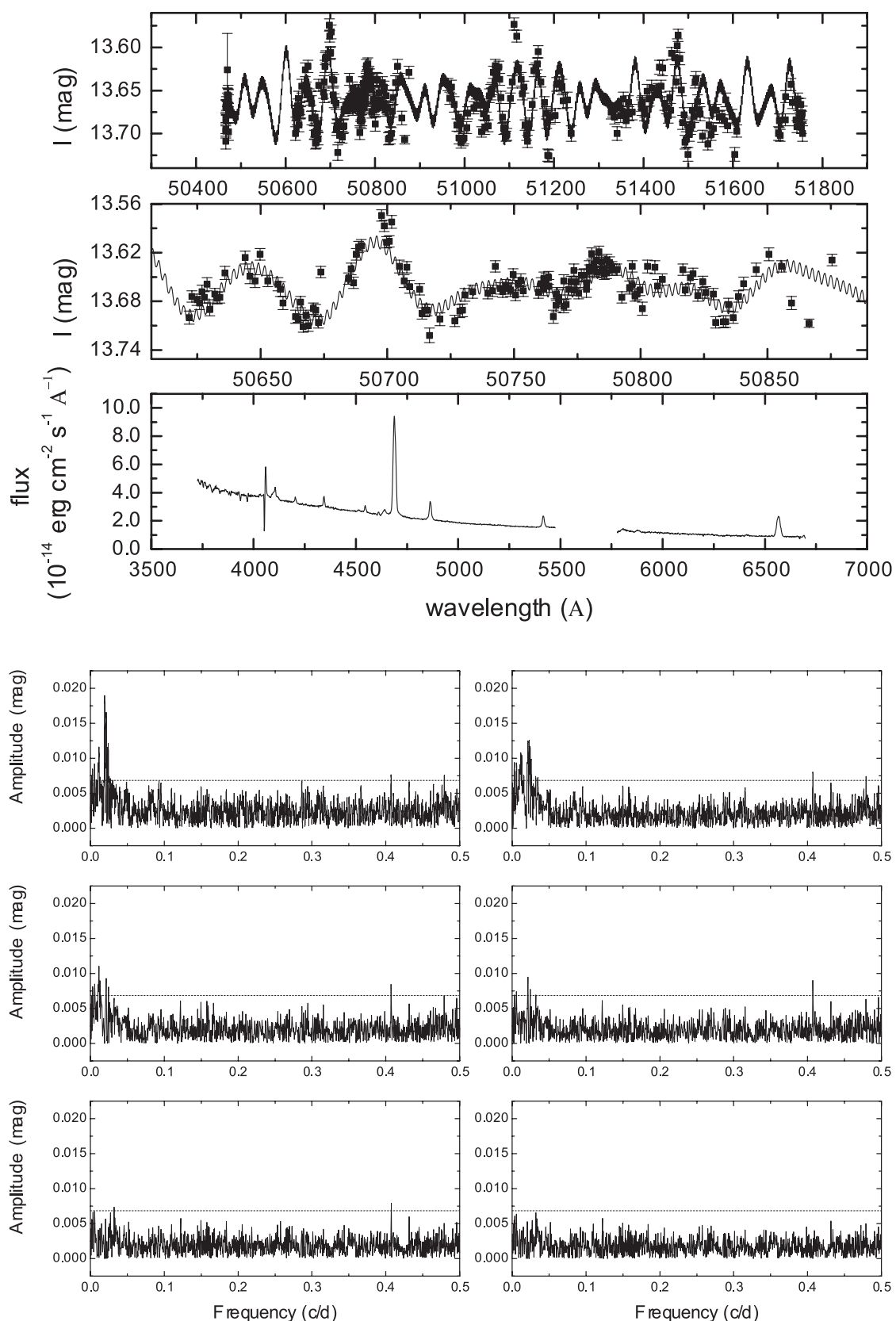

Fig. 8. a) OGLE light curve and the best-fitting function (upper), detail of the light curve and fit (middle) and spectrum (bottom) of SMC3-8. b) Fourier spectrograms illustrating the prewhitening process for SMC3-8. The dotted line indicates the significance level determined as explained in the text. The high frequency at $0.41 \mathrm{c} / \mathrm{d}$ is probably an artifact.

\subsubsection{OGLE00552027-7237101 (SMC3-14)}

This object was previously classified as the $\mathrm{H} \alpha$ emission line star MA93 836 (Meyssonnier \& Azzopardi 1993). The OGLE light curve shows complex periodic oscillations with a total amplitude of about 0.1 mag (Fig. 12a). M02 reported a period of $107 \pm 3$ days. The spectral type is compatible with A0 Ibe. The $\mathrm{H} \beta$ line shows an incipient emission at the red wing of the absorption line, i.e. a weak P-Cygni type profile, with maximum emission at velocity compatible with the $\mathrm{H} \alpha$ emission velocity (see Table 2). The Balmer absorption lines are blue-shifted by $190 \mathrm{~km} \mathrm{~s}^{-1}$ as regards the emission lines, which could be explained by unseen red emission in their absorption wings. The $\mathrm{H} \alpha$ equivalent width is $-18.5 \AA$. We observe strong absorption lines at $\lambda \lambda$ 5833.1, 5862.8, 5911.1, 5949.9, 5999.5, and 6081.1 (Fig. 12a). Using the velocity system of the $\mathrm{H} \alpha$ emission, we identified these lines as Fe I (except $\lambda$ 5833.1 $\AA$, which could not be identified). We also tried the velocity system of the blue lines, but it yielded inconsistent and unreliable identifications.

We found six frequencies, which fit the overall photometric behavior of the star relatively well, but do not reproduce some excursions in some epochs, revealing the presence of aperiodic or non-deterministic components in the light curve (Fig. 12a). We note that $\mathrm{F} 2=2 \mathrm{~F} 1$ for this star. F5 and F6 are not present in the OGLE-III data, hence they probably correspond to transient or spurious frequencies. The prewhitening process is illustrated in Fig. 12b. 
Table 4. Main lines detected in the WR star OGLE00504344-7327053 and their equivalent widths.

\begin{tabular}{lr}
\hline \hline Line & $E W(\AA)$ \\
\hline H $\delta+$ He II & -4.5 \\
He II $\lambda$ 4200 & -1.0 \\
H $\gamma+$ He II & -1.9 \\
He II $\lambda$ 4517 & -0.3 \\
He II $\lambda$ 4542 & -1.3 \\
N V $\lambda$ 4610 & -0.4 \\
N III 4642 & -2.0 \\
He II $\lambda$ 4686 & -43.4 \\
H $\beta+$ He II & -8.5 \\
He II $\lambda$ 5411 & -6.9 \\
C IV $\lambda$ 5802+5812 & -1.9 \\
He I $\lambda$ 5875 & -1.5 \\
H $\alpha+$ He II & -33.3 \\
\hline
\end{tabular}

\subsubsection{OGLE00574525-7235321 (SMC3-15)}

$U B V R$ photometry was presented by Massey (2002, its object SMC 41243). The OGLE light curve shows a slow simple oscillation (Fig. 13a). Our spectrum does not show emission lines, and it is compatible with a spectral type of B1 II-III. We found only one frequency, $0.00412 \pm 0.00132 \mathrm{c} / \mathrm{d}$, which prewhites the data well (Fig. 13b). This frequency is not found in OGLE-III data. Their very low amplitude ( $3 \mathrm{mmag})$ suggests that is an spurious detection. The period given by M02 is erroneous.

\subsubsection{OGLE00581258-7230485 (SMC3-16)}

This is the Be X-ray binary RX J0058.2-7231 (e.g. Schmidtke et al. 2003, hereafter S03). The OGLE light curve shows a series of low-amplitude ( $\Delta I \sim 0.05 \mathrm{mag}$ ) outbursts every 60-days and a sustained increase in the mean brightness (Fig. 14a). S03 suggest that the brightening may be related to changes in the Be star disc at periastron, assuming that the neutron star is in an eccentric orbit. They noted the narrow eclipse at maximum light, which explained as the eclipse of an excited region in the disc, by the B-type star, and provided the following ephemeris for minimum light (subtracting the long-term tendency): $P_{\text {mean }}=$ 59.72 days and $T_{0}=$ MJD 50030 . They also report evidence that the period may be decreasing at a rate of $\sim 0.01$ days per cycle. Our analysis of OGLE data confirms the narrow eclipse at maximum and shows that the period is changing (Fig. 14a); furthermore, the joint analysis of MACHO and OGLE data, covering 74 orbital cycles, indicates a quasicyclic period change with a time scale of variability of $\sim 1200$ days (Fig. 14a). Our spectra were taken during the bright phase, at phases 0.30 (blue) and 0.33 (red). At these phases we observe $\mathrm{H} \alpha$ and $\mathrm{H} \beta$ emission, with equivalent widths of $-15.5 \AA$ and $-1.7 \AA$, respectively. The continuum drops dramatically in a lapse of two days (Fig. 14a), more than the $\$ 0.1 \mathrm{mag}$ expected from the light curve. We have doubts about the reality of this finding, since it could indicate a drop in the atmospheric transparency during our observation obtained in the red wavelength range, probably by the passage of a thin cloud. However, our records indicate that the weather was good and the sky clear that night, so we have no argument to support this explanation.

The full width at zero intensity of the $\mathrm{H} \alpha$ line is $976 \mathrm{~km} \mathrm{~s}^{-1}$. S03 predicted, according to their proposed eccentric binary model, the appearance of He II 4686 during the bright phase, and also the strengthening of the $\mathrm{H} \alpha$ emission. However, we observed neither He II in our spectra nor stronger $\mathrm{H} \alpha$ emission. Comparing with the emission line data given by S03 (based on two spectra taken at phases 0.04 and 0.70 ), we conclude that the $\mathrm{H} \alpha$ equivalent width seems to be constant during the 60-day cycle, while their width does not. The three available measurements suggest that the $\mathrm{H} \alpha$ emission width is maximum at minimum light, just before starting the outburst, and then decreases during the slow outburst decline. This could be explained as the formation of a reservoir of high-velocity gas around the compact object prior to outburst. The optical outburst could be due to the accretion of this gas reservoir onto the compact object and the subsequent reprocessing of high-energy photons in the Be-star envelope. More observations are needed to confirm this scenario. We determined a spectral type of B0 III e from the appearance of the blue absorption features in the spectra.

We removed the long-term tendency of the light curve by fitting a straight line to the data, and worked with the residuals. The four frequencies we found (Table 3) reproduce the shape of the light curve well, but only F1 and F2 are present in OGLE-III data, and they probably represent the fitting to a non-sinusoidal light curve rather than true physical frequencies (Fig. 14a). The prewhitening process is illustrated in the periodograms of Fig. 14b.

The period change in this binary could be compared with the X-ray variability predicted by recent models of Be X-ray binaries by Hayashaki \& Okazaki $(2005,2004)$. X-ray variability could modulate the optical light curve through re-processing of high energy photons by the Be star envelope. The aforementioned authors studied the long-term evolution of accretion discs around the neutron star in Be X-ray binaries using a three-dimensional, smoothed particle hydrodynamics (SPH) code. They showed that a time-dependent accretion disc is formed around the neutron star and that the disc has a one-armed spiral structure that is induced by a phase-dependent mass transfer from the Be disc. Hayashaki \& Okazaki (2005) find that, after the disc is fully developed, the peak mass-accretion rate is distributed at phases around apoastron, which they interpret as evidence that the mass accretion rate is basically caused by an inward propagation of the one-armed spiral wave. According to their model, the time scale of variability for the X-ray luminosity peak is around 24 orbital cycles. This number is remarkably close to our number (1200 days corresponds to 20 orbital cycles). However, the distribution of the luminosity peak in the models of Hayashaki \& Okazaki amounts to a few tenths of the orbital cycle, much more than our detected variations of a few percents of the orbital cycle. The Hayashaki \& Okazaki simulations also show the interesting result that a double-peaked X-ray maximum is observed when the disc is growing to maximum size. In contrast to the persistent double maxima observed in the optical light curve of RX J0058.2-7231, the structure seen in the simulations disappears when the disc is fully developed, after a few tenths of the orbital cycles.

\subsubsection{OGLE01000078-7255229 (SMC3-18)}

This is the emission line object AzV 241 or Lin 363 (Azzopardi \& Vigneau 1982; Lindsay 1961). The OGLE light curve shows complex, presumably periodic, variability (Fig. 15a). M02 report a period of $35 \pm 3$ days. Our spectrum shows $\mathrm{H} \beta$ emission with an equivalent width of $-2.0 \AA$, with the other Balmer lines probably partly filled by emission. Weak helium lines are also visible in the spectrum. We determined a spectral type of B8-9IIIe. Our search for frequencies yielded five significant frequencies 
HJD - 2400000
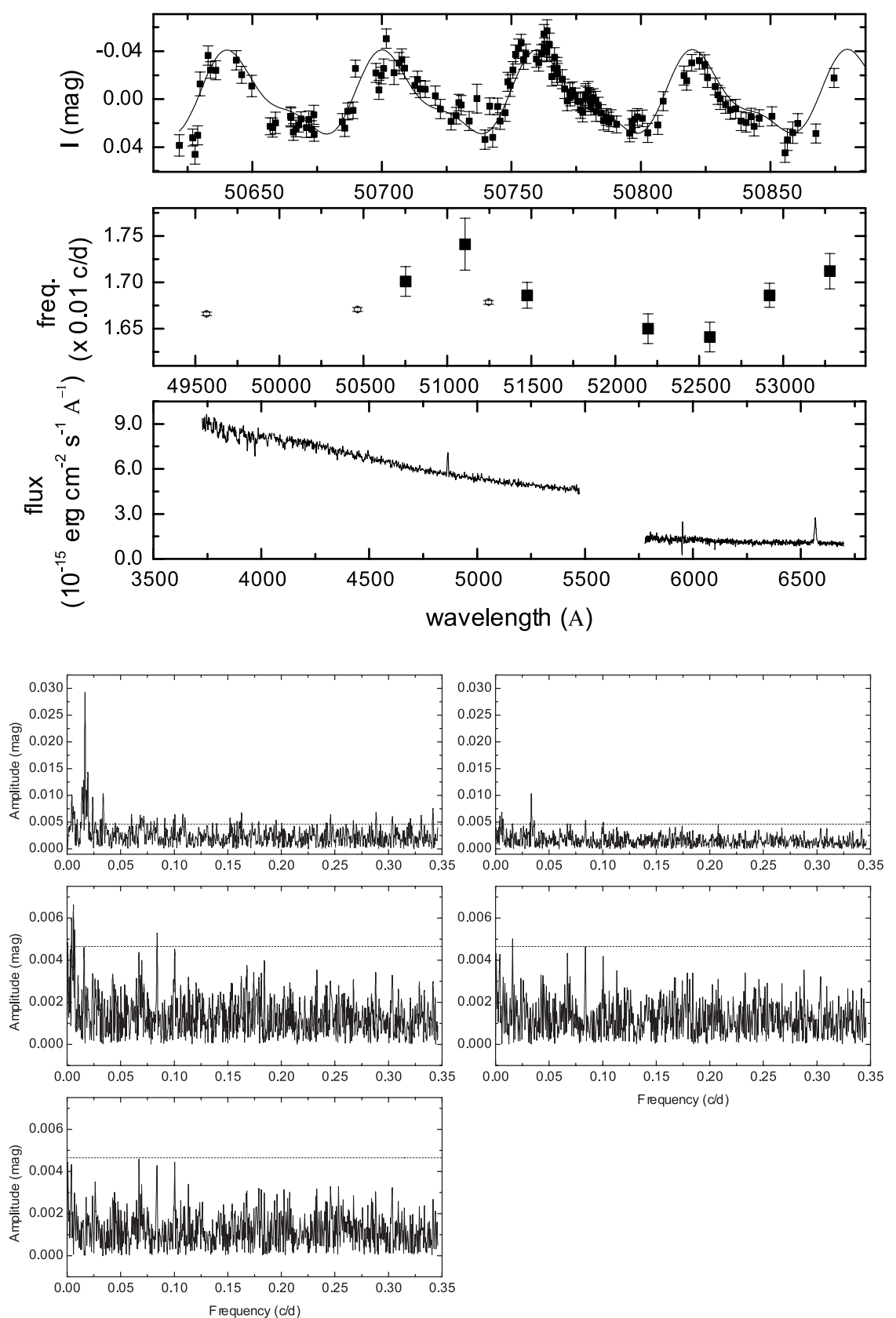

Fig. 14. a) Detail of the light curve and fit (upper), the frequency as a function of time (middle) and the spectrum (bottom) of SMC3-16. In the middle panel filled squares correspond to our data and open circles to data from Schmidtke et al. (2003). b) Fourier spectrograms illustrating the prewhitening process for SMC3-16. The dotted line indicates the significance level determined as explained in the text.

for this star (Table 3). In general these frequencies fit the overall shape of the light curve well, but several outliers at different epochs indicate aperiodic components in the data. The only frequency of this set that survives during the OGLE-III period is F1. The prewhitening process is shown in the periodograms of Fig. 15b.

\subsubsection{OGLE01045121-7246469 (SMC3-20)}

This is the emission line object AzV 366 or Lin 437 (Azzopardi \& Vigneau 1982; Lindsay 1961). The OGLE light curve shows periodic variability (Fig. 16a). M02 reported a periodicity of $44 \pm 3$ days. The spectrum shows prominent $\mathrm{H} \alpha$ and
$\mathrm{H} \beta$ emission lines and the spectral type is $\mathrm{B} 1 \mathrm{Ib}$-IIe. The $\mathrm{H} \alpha$ equivalent width is $-46.2 \AA$. Only one frequency is found in our analysis, $0.02249 \pm 0.00006 \mathrm{c} / \mathrm{d}$, which is also observed in the OGLE-III dataset, confirming the M02 report. Aperiodic activity is also visible in some epochs (see outliers in Fig. 16a). The Fourier periodograms are shown in Fig. $16 \mathrm{~b}$.

\subsubsection{OGLE01045299-7159188 (SMC3-21)}

This is the emission line star AzV 364, also named MA93 1492 (Azzopardi \& Vigneau 1982; Meyssonnier \& Azzopardi 1993). The OGLE light curve shows a periodic variability of $44 \pm$ 3 days (Fig. 17a, M02). Our spectrum shows weak $\mathrm{H} \alpha$ emission 
Table 5. 2MASS $J$ magnitude and colors (in mag) for the stars of this paper. The $J-H$ color expected for the spectral type, according to Koornneef (1983) and Winkler (1997, for B-type stars), is also shown, along with the estimated color excess.

\begin{tabular}{lcccccccc}
\hline \hline star-id & $J$ & error & $J-H$ & error & $H-K$ & error & $(J-H)_{\text {sp }}$ & $E(J-H)$ \\
\hline SMC3-1 & 13.54 & 0.04 & 0.20 & 0.07 & 0.07 & 0.06 & 0.16 & 0.05 \\
SMC3-2 & 13.74 & 0.05 & 0.32 & 0.09 & 0.11 & 0.09 & -0.02 & 0.34 \\
SMC3-3 & 14.25 & 0.02 & 0.36 & 0.04 & 0.09 & 0.07 & 0.15 & 0.21 \\
SMC3-4 & - & - & 0.17 & 0.04 & 0.18 & 0.05 & 0.22 & -0.05 \\
SMC3-5 & 14.70 & 0.05 & 0.17 & 0.09 & 0.04 & 0.38 & -0.03 & 0.20 \\
SMC3-6 & 13.69 & 0.02 & 0.18 & 0.04 & 0.08 & 0.05 & 0.10 & 0.08 \\
SMC3-7 & 13.19 & 0.03 & 0.20 & 0.04 & 0.03 & 0.05 & 0.20 & -0.00 \\
SMC3-8 & 13.59 & 0.04 & 0.07 & 0.06 & 0.13 & 0.06 & - & - \\
SMC3-9 & 13.24 & 0.04 & 0.21 & 0.07 & 0.22 & 0.07 & -0.09 & 0.30 \\
SMC3-10 & - & - & -0.19 & 0.14 & 0.21 & 0.19 & - & - \\
SMC3-12 & 13.85 & 0.03 & 0.13 & 0.05 & 0.07 & 0.06 & 0.09 & 0.04 \\
SMC3-14 & 14.43 & 0.04 & 0.13 & 0.05 & 0.04 & 0.09 & 0.08 & 0.05 \\
SMC3-15 & 14.18 & 0.03 & -0.14 & 0.08 & 0.08 & 0.10 & -0.09 & -0.05 \\
SMC3-16 & 14.55 & 0.04 & 0.11 & 0.08 & 0.26 & 0.09 & -0.10 & 0.21 \\
SMC3-18 & 13.94 & 0.04 & 0.14 & 0.04 & 0.12 & 0.06 & -0.02 & 0.16 \\
SMC3-20 & 13.27 & 0.03 & 0.09 & 0.06 & 0.24 & 0.06 & -0.07 & 0.16 \\
SMC3-21 & 14.44 & 0.03 & 0.09 & 0.05 & -0.03 & 0.09 & -0.08 & 0.17 \\
\hline
\end{tabular}

and $\mathrm{H} \beta$ partly filled by emission. The $\mathrm{H} \alpha$ line has an equivalent width of $-12 \AA$. We derived a spectral type of B2 IIIe. We found four significant frequencies (Table 3), but only F1, those found by M02, survives the stability test. The Fourier periodograms are shown in Fig. 17b.

\subsection{Infrared photometry and color excess}

In this section we present and discuss the infrared photometry extracted from the 2MASS catalogue using the VizieR on-line query form (Ochsenbein et al. 2000). We estimated the total $J-H$ color excess using the expected colors for the spectral types and luminosity classes given in Sect. 3.1. The results are shown in Table 5. We did not attempt to determine absolute magnitudes from 2MASS photometry, since the large amount of infrared free-free and free-bound emission for stars with gaseous envelopes is hard to estimate with our data.

In Fig. 18 we show the color-color diagram for all objects, along with the position for classical Be stars and the main sequence and supergiant galactic tracks. We observe that the stars showing multiple periods and those with transient periodicities lie close to the main sequence and supergiant tracks, whereas those with single stable periods and the eclipsing and ellipsoidal binaries show much more reddening in general. The star SMC3-5 (those with the largest error bar in the $H-K$ color in Fig. 18) has a flag in the 2MASS catalogue indicating photometric confusion probably by a nearby companion, therefore its infrared photometry should be taken with caution.

\section{Discussion}

In the preceding section we have given a detailed discussion of the observed properties of each star in our sample. In this section, we focus on the general picture we can extract from this study. For this reason, in the following we exclude the objects SMC3-8 and SMC3-16, as well as the eclipsing and ellipsoidal variables, which were extensively discussed in the previous section, and will focus our attention on the remaining objects of our sample.

A general pattern seems to arise from the analysis in Sect. 3. We identify three general groups of stars according to their photometric and spectroscopic properties. The first group corresponds to those stars showing transient periods; they are

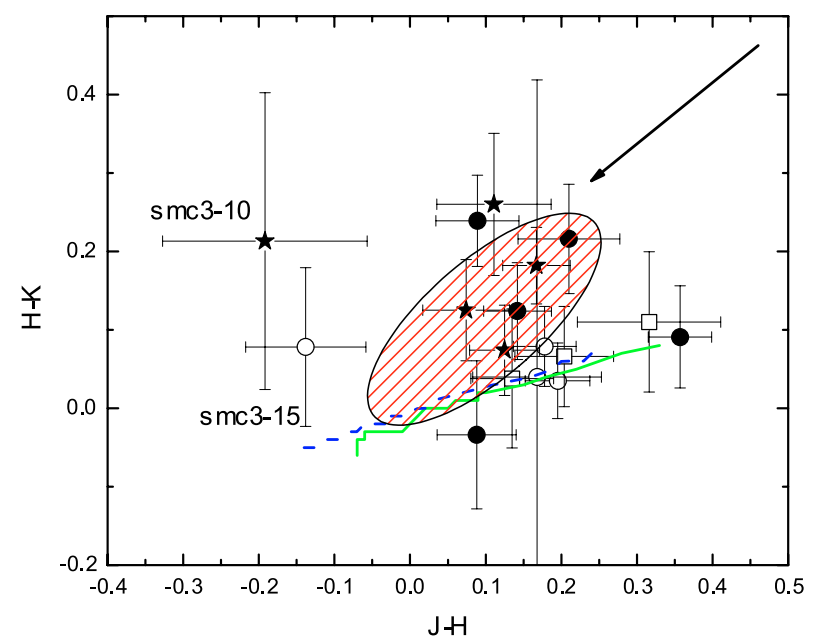

Fig. 18. The color-color diagram for the stars studied in this paper. The objects showing transient periods are indicated by open circles, those with single stable periods by filled circles, those with multiple periods by open squares, and the ellipsoidal and eclipsing binaries are indicated by asterisks. The colors for galactic main sequence and supergiant O9-G0 stars are also shown by the upper and bottom lines, respectively (Koornneef 1983). The dashed oval indicates the region occupied by the classical Be stars of Dougherty et al. (1994). The arrow shows the reddening vector for $R_{V}=3.3$ and a visual extinction of 2.5 mag.

4 non-emission stars showing very small photometric amplitudes, except SMC3-6 displaying an amplitude of 0.05 mag. The second group consists of stars showing only one stable frequency; it corresponds to 4 giant Be stars and one FOIb-II star. Finally, the third group displays multiperiodicities and consists of 2 A-type and 1 B7-9e stars.

It is possible that the variability for the first two groups arises from emitting gaseous envelopes and not from the star itself. Transient objects could be explained as those having low density, highly variable, low-emission envelopes. This should explain the low amplitude of the variability and their transient nature. It is well known that episodes of mass ejection in Be stars produce brightness variability. If these ejections consist of gas blobs taking angular momentum away from the star, they should 
rotate producing a quasi-periodic pattern of brightness before dissolving in the interstellar medium. This view requires gas ejections from the stellar surface, a process normally associated to rapid rotation, which should work in the B-A stars of our sample (given the required conditions), but probably not for SMC3-7 having a spectral type of F4 IV. This explanation is supported by the finding that a much larger fraction of massive stars at lower $Z$ environments (like that found in the $S M C$ ) reaches break-up velocities. The reason is that at low $Z$, mass loss is smaller and the removal of angular momentum during evolution is much weaker (Maeder \& Meynet 2001).

On the other hand, well-developed envelopes, which are a signature of classical Be-type stars, could be responsible for the stable, long-term variability observed in most Be stars of Table 2 . This variability could be explained as changing aspects of an elliptical envelope in a binary system (by precession, for instance), or it eventually could be caused by ellipsoidal variability masked by additional phenomena related to the formation and dissipation of the Be-star envelope. The amplitudes of the variations are in principle compatible with ellipsoidal variability. Therefore, these objects are serious candidates for binarity. While Be star binaries amount to $20 \%$ of the total Be-star population in our galaxy (e.g. Porter \& Rivinius 2003), a corresponding figure for the $S M C$ has not yet been established. Therefore, these objects are ideal targets for RV studies aimed at confirming their binary nature and at testing binary formation models in different metallicity environments (e.g. de Loore \& Vanbeveren 1995).

Finally, the last group of variables in this study corresponds to stars showing multiple periods. As noted before, the time scale of variability does not fit any known stellar pulsation mechanism. However, all of them are emission line stars, so the variability could be associated to the envelope. Binarity could be the right scenario for these objects, and future time-resolved radial velocity studies are needed to check this view.

\section{Conclusions}

In this paper we have presented a photometric and spectroscopic study of 17 bright blue and yellow variable stars in the direction of the SMC. These stars were previously classified type-3 by Mennickent et al. (2002); i.e., they correspond to photometrically periodic variables, Be star candidates, whose nature was not completely clear. During our analysis, we detected peculiar behavior in several light curves and interesting new features in the spectra of these stars. We summarize our findings as follows:

- We find a beating phenomenon in the star OGLE004454667328029 (SMC3-2), which could be explained by pulsation and misaligned orbital-pulsational axes in a binary system. However, the physical basis for the putative pulsation (of period about 15 days) remains unclear. This star also shows anomalous absorption lines attributed to lowexcitation metallic ions.

- We find a variable photometric period in the Be X-ray binary OGLE00581258-7230485 (SMC3-16) that could be linked to the recent discovery of a variable phase of maximum X-ray light in highly eccentric Be X-ray binaries. Alternatively, we could interpret the changing period as evidence of a tilted disc swinging around the apsidal line.

- All our stars are very likely members of the SMC, except OGLE00502564-7258071 (SMC3-7), which was classified F4IV and turned out to be a possible member of the Milky-Way halo.
- We determine spectral types and radial velocities for most of our objects, and absolute magnitudes and colors for some of them. Most stars are B-F giants or supergiants showing emission line spectra.

- We find a new long-period interacting eclipsing binary (OGLE00463376-7312043, SMC3-4) showing emission lines in its spectrum, and two new ellipsoidal variables, OGLE00510759-7326366 (SMC3-10) and OGLE00535922-7235089 (SMC3-12).

- We have found transient photometric periodicity in 4 objects. We suggest that they could indicate the activity of a changing gaseous envelope around rapidly rotating stars.

- We find long-term stable periods in 4 Be stars that could be associated to binarity. The population of Be star binaries in the $S M C$ is poorly understood, so these objects are priority targets for future spectroscopic monitoring.

- We find multiple periods in three early-type emission line stars, OGLE00433691-7326377 (SMC3-1), OGLE00445466-7328029 (SMC3-2), and OGLE005520277237101 (SMC3-14). The variability of these objects could be partly explained by binarity.

Our results confirm the suspicion by M02 that "type-3" variables harbor a mix of different kind of stars, not necessarily related to the $\mathrm{Be}$ star phenomenon. We note the absence of Herbig Ae/Be objects in our sample and the presence of several binaries. In a forthcoming paper we will discuss a similar sample of type- 3 stars in the $L M C$, making a comparative analysis with the type-3 variables found in the $S M C$ and discussed in this paper.

Further studies are clearly needed to improve our knowledge about these objects. For instance, monitoring radial velocities over extended periods of time should be desirable to test the binary hypothesis. To fully understand the variable stars discovered by programs like OGLE and MACHO, time-resolved spectroscopic and photometric data must be analyzed together. Part of our future research programs will focus on this goal.

Acknowledgements. We acknowledge the anonymous referee who helped us to improve the first version of this article. R.M. acknowledges support by Fondecyt grant 1030707. R.M., W.G. and G.P. acknowledge support from the Chilean FONDAP centre for Astrophysics 15010003. B. Sabogal acknowledges support by Programa MECE Educación Superior UCO0209. This research has made use of the SIMBAD database, operated at the CDS, Strasbourg, France. This publication makes use of data products from the Two Micron All Sky Survey, which is a joint project of the University of Massachusetts and the Infrared Processing and Analysis centre, funded by the National Aeronautics and Space Administration and the National Science Foundation.

\section{References}

Aerts, C., \& Kolenberg, K. 2005, A\&A, 431, 615

Ahumada, A. V., Clariá, J. J., Bica, E., \& Dutra, C. M. 2002, A\&A, 393, 855

Azzopardi, M. \& Vigneau, J. 1982, A\&A, 50, 291

Baldry, I. K., Kurtz, D. W., \& Bedding, T. R. 1998, MNRAS, 300, L39

Breger, M., et al. 1993, A\&A, 271, 482

Cox, A. N. 2000, Allen's Astrophysical Quantities, Springer, AIP Press Crowther, P. A. 2000, A\&A, 356, 191

de Wit, W. J., Beaulieu, J.-P., Lamers, H. J. G. L. M., Lesquoy, E., \& Marquette, J.-B. 2003, A\&A, 410, 199

de Wit, W. J., Beaulieu, J. P., Lamers, H. J. G. L. M., Coutures, C., \& Meeus, G. 2005, A\&A, 432, 619

de Loore, C., \& Vanbeveren, D. 1995, A\&A, 304, 220

Didelon, P. 1982, A\&AS, 50, 199

Dougherty, S. M., Waters, L. B. F. M., Burki, G., et al. 1994, A\&A, 290, 609

Foellmi, C., Moffat, A. F. J., \& Guerrero, M. A. 2003, MNRAS, 338, 360

Frank, J., King, A., \& Raine, D. J. 2002, Accretion Power in Astrophysics, third edition, Cambridge Astrophysics Series, 21

Hayasaki, K., \& Okazaki, A. T. 2004, A\&A, 350, 971

Hayasaki, K., \& Okazaki, A. T. 2005, A\&A, 360, L15 
Henize, K. G. 1956, ApJS, 2, 315

Koornneef, J. 1983, A\&A, 128, 84

Lenz, P., \& Breger, M. 2005, CoAst, 146, 53

Lindsay, E. M. 1961, AJ, 66, 169

Kiss, L. L., Szatmáry, K., Szabó, G., \& Mattei, J. A. 2000, A\&AS, 145, 283

Maeder, A., \& Meynet, G. 2001, A\&A, 373, 555

Massey, P. 2002, AJSS, 141, 81

Massey, P., \& Duffy, A. S. 2001, ApJ, 550, 713

Mennickent, R. E., Vogt, N., \& Sterken, C. 1994, A\&AS, 108, 237

Mennickent, R. E., Pietrzyński, G., Gieren, W., \& Szewczyk, O. 2002, A\&A, 393,887

Mennickent, R. E., Assmann, P., Pietrzyński, G., \& Gieren, W. 2003, in Proc. of the workshop, The Light-Time Effect in Astrophysics, ed. C. Sterken, ASP conf. Ser., 292, 89

Meyssonnier, N., \& Azzopardi, M. 1993, A\&AS, 102, 451

Moffat, A. F. J. 1988, ApJ, 330, 766
Munari, U., \& Zwitter, T. 1997, A\&A, 318, 269

Ochsenbein, F., Bauer, P., \& Marcout, J. 2000, A\&AS, 143, 23

Pamyatnykh, A. A. 1999, AcA, 49, 119

Porter, J. M., \& Rivinius, T. 2003, PASP, 115, 1153

Reed, M. D., \& Brondel, B. J. 2005, ASPC, 333, 228

Sabogal, B. E., Mennickent, R. E., Pietrzyński, G., \& Gieren, W. 2005, MNRAS, 361,1055

Schmidtke, P. C., Cowley, A. P., \& Levenson, L. 2003, AJ, 126, 1017

Sterken, C. 2005, ASPC, 335, 3

Stock, J., \& Stock, J. M. 1999, RMxAA, 35, 143

Stock, M. J., Stock, J., García, J., \& Sánchez, N. 2002, RMxAA, 38, 127

Winkler, H. 1997, MNRAS, 287, 481

Zaritsky, D., Harris, J., Thompson, I. B., Grebel, E. K., \& Massey, P. 2002, AJ, 123,855

Zebrun, K., et al. 2001, AcA, 51, 317 
R. E. Mennickent et al.: Long-period variables towards the SMC, Online Material $p 1$

\section{Online Material}


R. E. Mennickent et al.: Long-period variables towards the SMC, Online Material $p 2$

HJD - 2,400,000
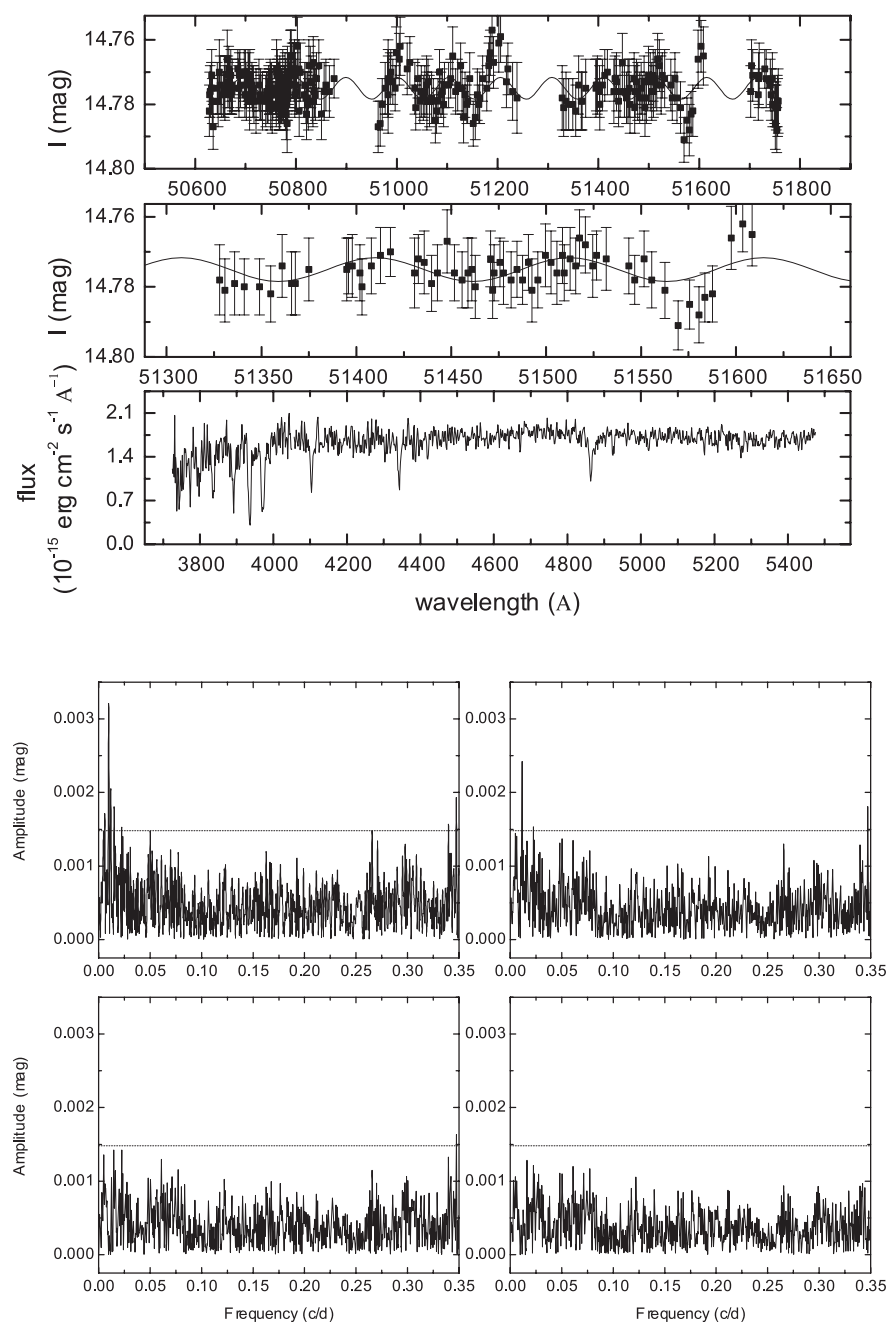

Fig.3. a) OGLE light curve and the best-fitting function (upper), detail of the light curve and fit (middle) and spectrum (bottom) of SMC3-3. b) Fourier spectrograms illustrating the prewhitening process for SMC3-3. The dotted line indicates the significance level determined as explained in the text.
HJD $-2,400,000$
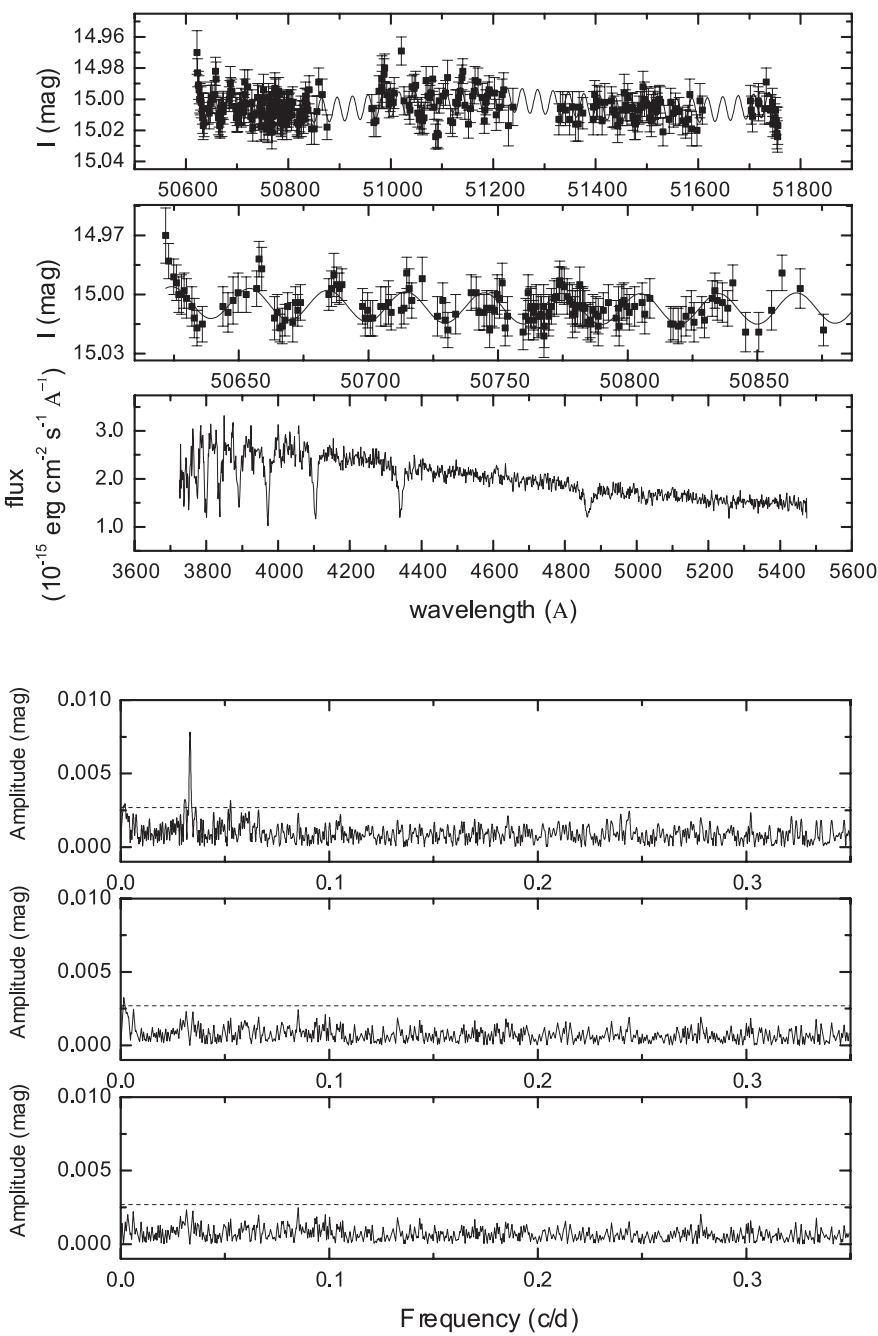

Fig. 5. a) OGLE light curve and the best fitting function (upper), detail of the light curve and fit (middle) and spectrum (bottom) of SMC3-5. b) Fourier spectrograms illustrating the prewhitening process for SMC3-5. The dotted line indicates the significance level determined as explained in the text. 
R. E. Mennickent et al.: Long-period variables towards the SMC, Online Material $p 3$
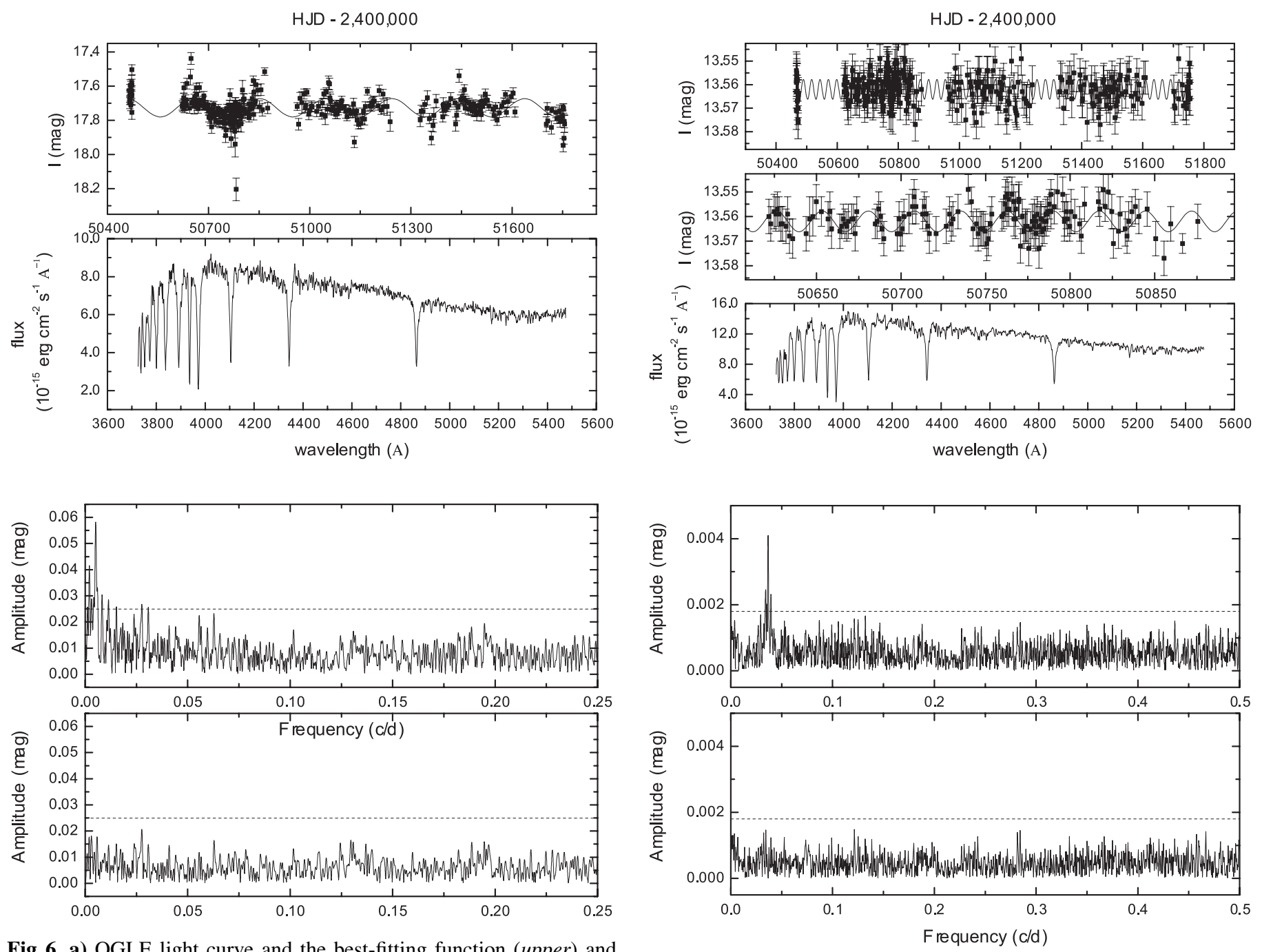

Fig. 6. a) OGLE light curve and the best-fitting function (upper) and spectrum (bottom) of SMC3-6. b) Fourier spectrograms illustrating the prewhitening process for SMC3-6. The dotted line indicates the significance level determined as explained in the text.

Fig. 7. a) OGLE light curve and the best-fitting function (upper), detail of the light curve and fit (middle) and spectrum (bottom) of SMC3-7. b) Fourier spectrograms illustrating the prewhitening process for SMC3-7. 
R. E. Mennickent et al.: Long-period variables towards the SMC, Online Material $p 4$

HJD - 2,400,000
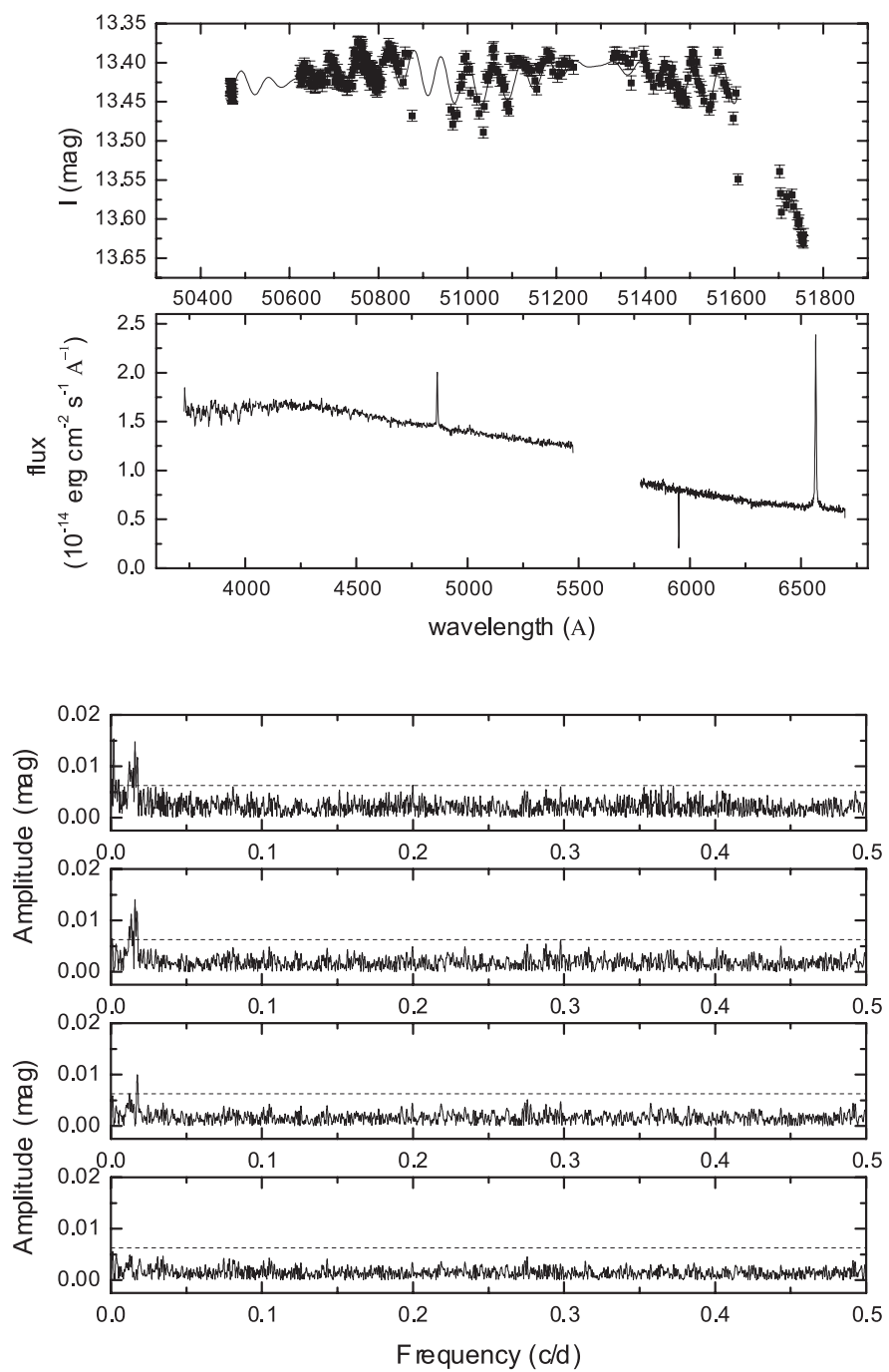

Fig. 9. a) OGLE light curve and the best-fitting function (upper) and spectrum (bottom) of SMC3-9. b) Fourier spectrograms illustrating the prewhitening process for SMC3-9. The dotted line indicates the significance level determined as explained in the text.
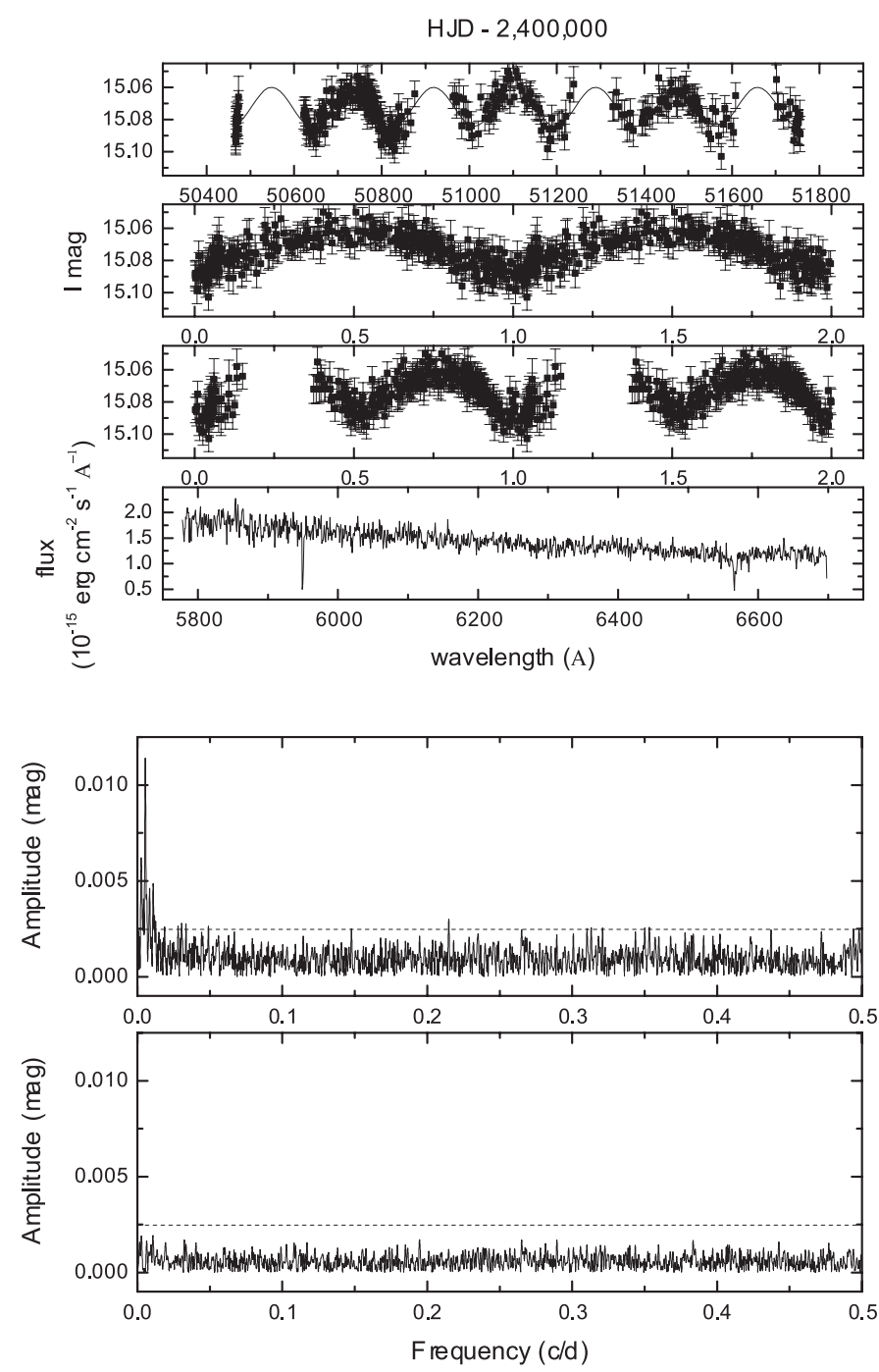

Fig. 10. a) OGLE light curve and the best-fitting function (upper), the light curve folded with the period 184.84 days and 369.69 days (middle panels) and spectrum (bottom) of SMC3-10. We suggest that the second periodicity is the right one. b) Fourier spectrograms illustrating the prewhitening process for SMC3-10. The dotted line indicates the significance level determined as explained in the text. 
R. E. Mennickent et al.: Long-period variables towards the SMC, Online Material p 5

HJD - 2,400,000
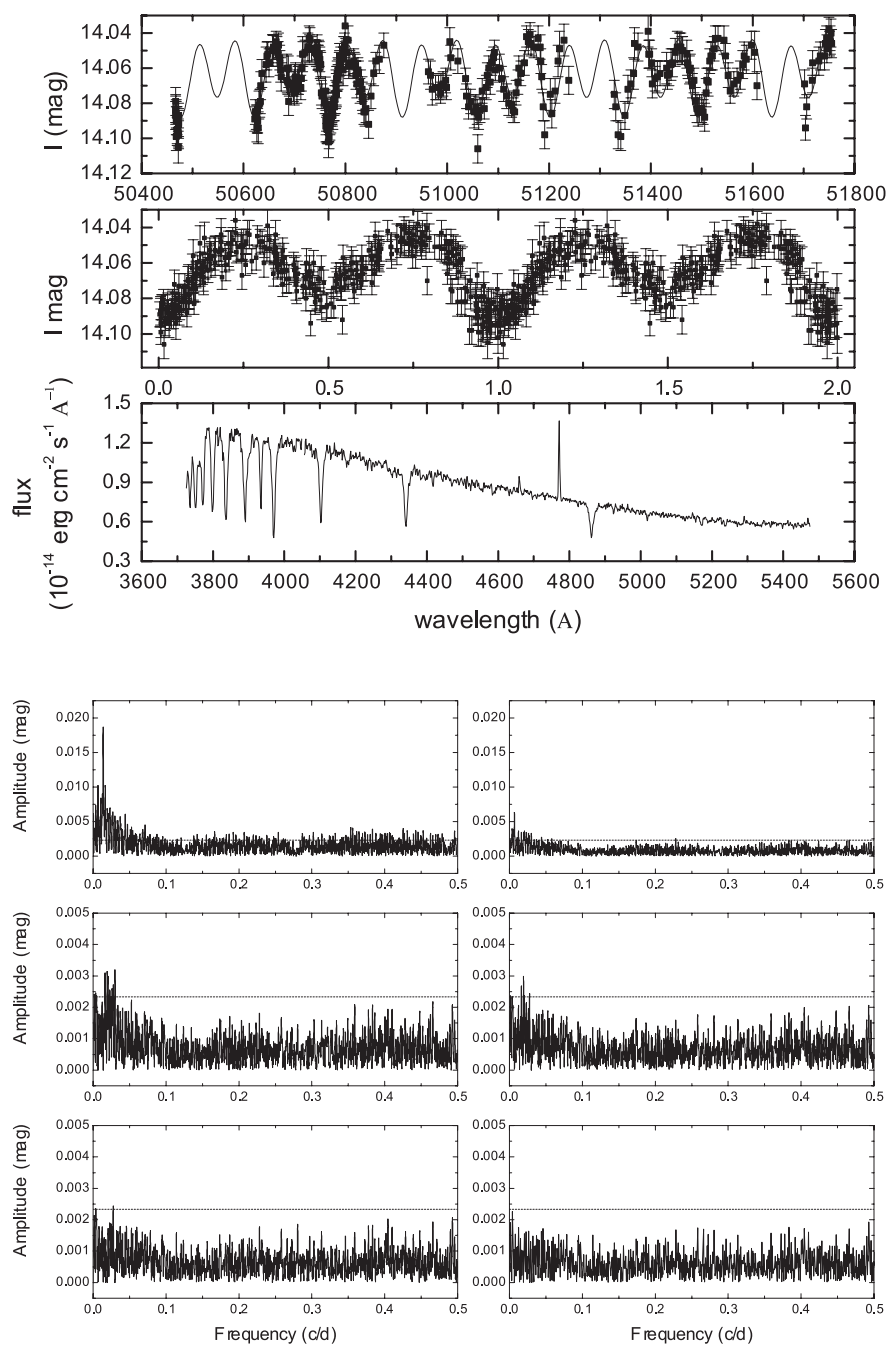

Fig. 11. a) OGLE light curve and the best-fitting function (upper), light curve folded with the orbital period (middle, the $x$-axis units are cycles) and spectrum (bottom) of SMC3-12. The peaks at $4659 \AA$ and $4772 \AA$ are artifacts not removed by the reduction process; they probably correspond to hits of cosmic rays. b) Fourier spectrograms illustrating the prewhitening process for SMC3-12. The dotted line indicates the significance level determined as explained in the text.
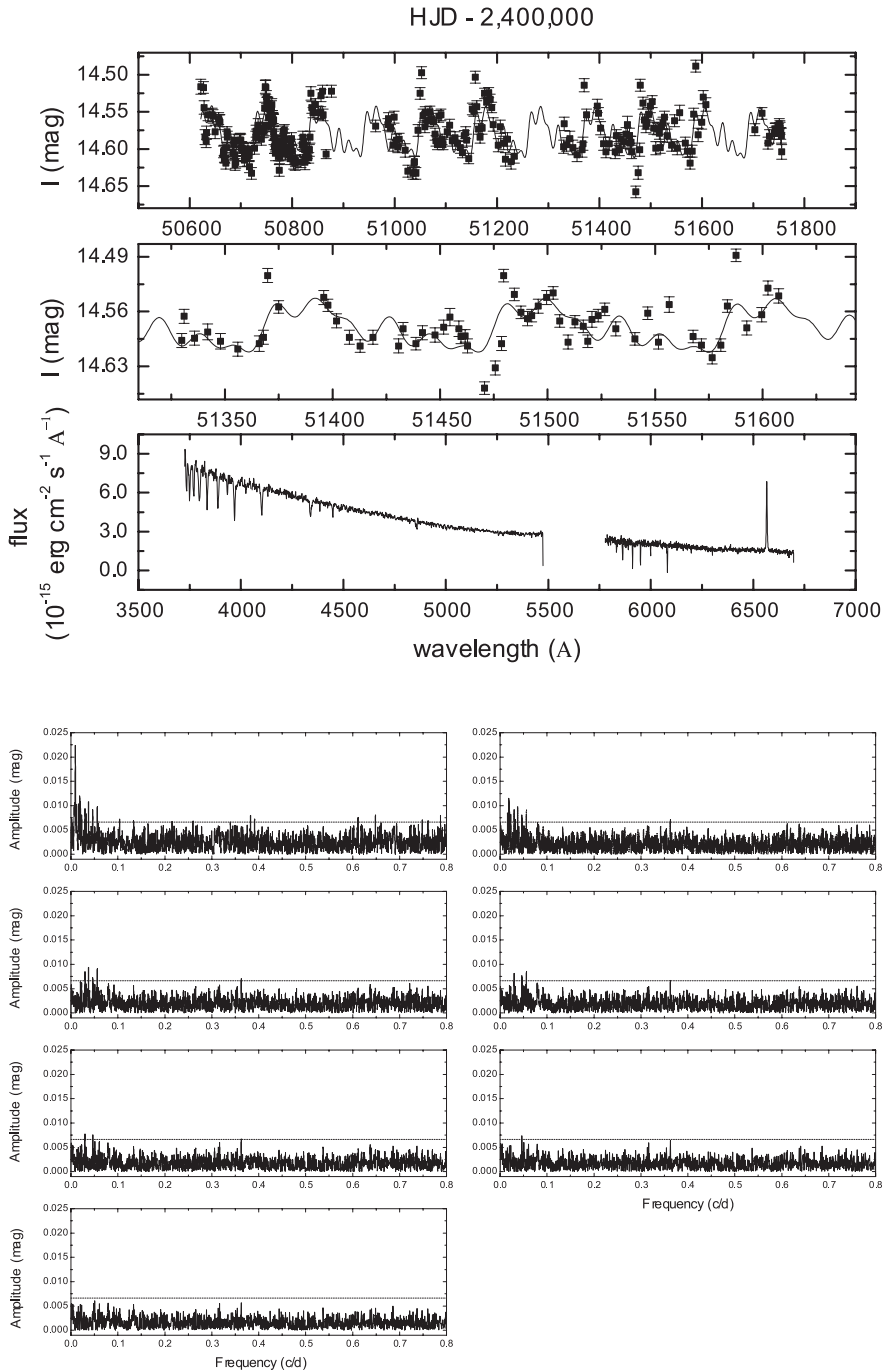

Fig. 12. a) OGLE light curve and the best-fitting function (upper), detail of the light curve and fit (middle) and spectrum (bottom) of SMC3-14. b) Fourier spectrograms illustrating the prewhitening process for SMC3-14. The dotted line indicates the significance level determined as explained in the text. 
R. E. Mennickent et al.: Long-period variables towards the SMC, Online Material $p 6$
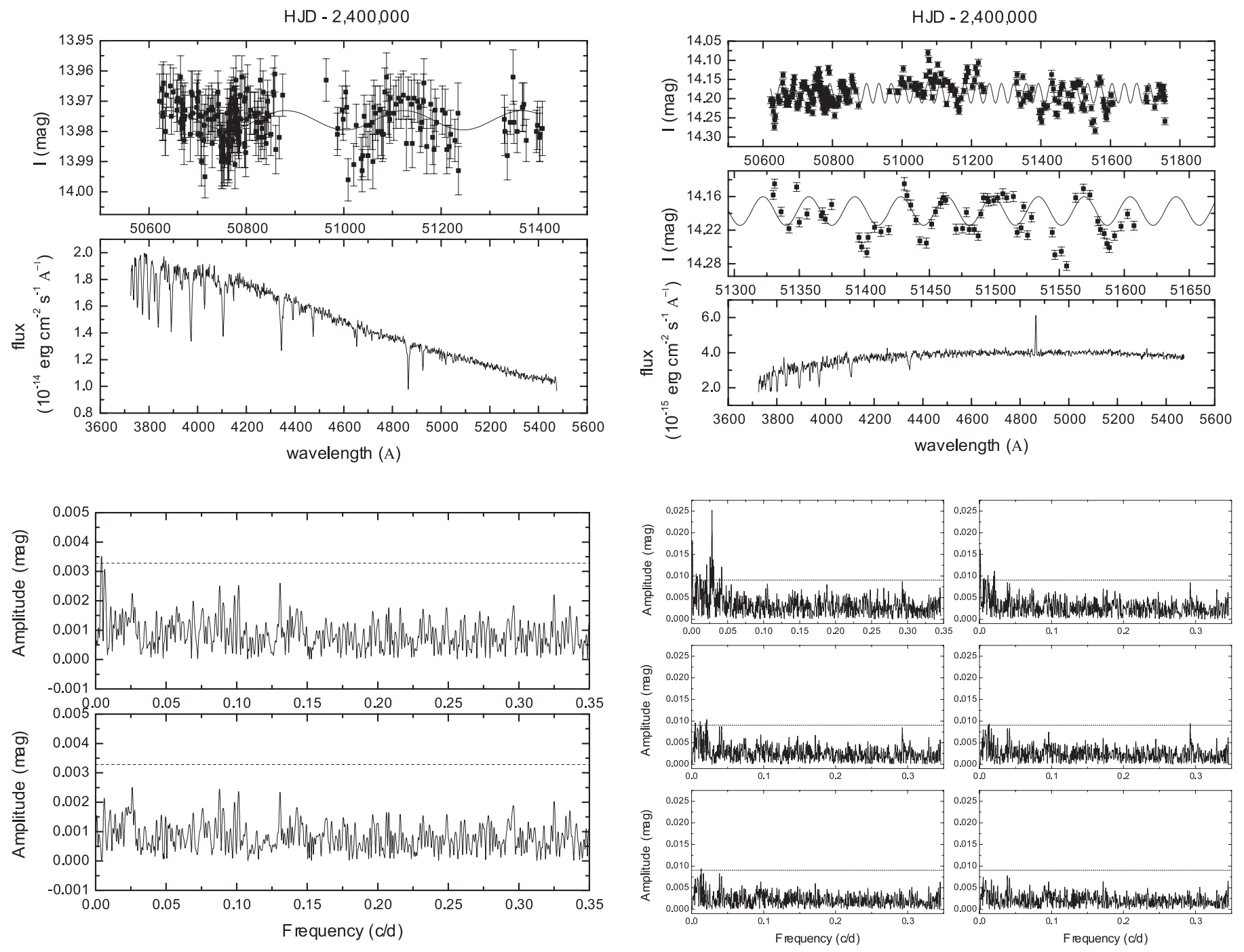

Fig. 13. a) OGLE light curve and the best-fitting function (upper) and spectrum (bottom) of SMC3-15. b) Fourier spectrograms illustrating the prewhitening process for SMC3-15. The dotted line indicates the significance level determined as explained in the text.

Fig. 15. a) OGLE light curve and the best-fitting function (upper), detail of the light curve and fit (middle) and spectrum (bottom) of SMC3-18. b) Fourier spectrograms illustrating the prewhitening process for SMC3-18. The dotted line indicates the significance level determined as explained in the text. 
R. E. Mennickent et al.: Long-period variables towards the SMC, Online Material $p 7$

HJD - 2,400,000
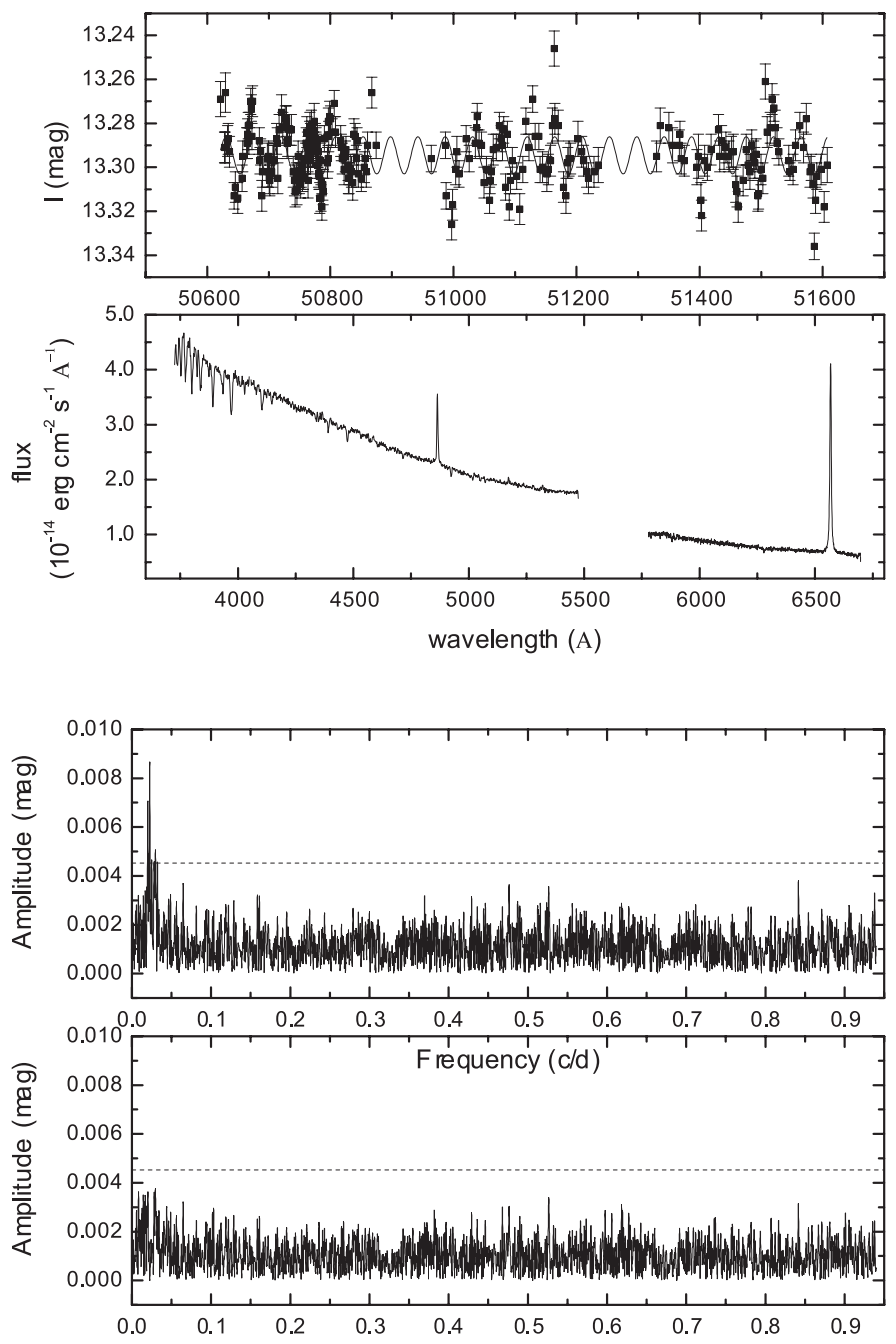

Fig. 16. a) OGLE light curve and the best-fitting function (upper) and spectrum (bottom) of SMC3-20. b) Fourier spectrograms illustrating the prewhitening process for SMC3-20. The dotted line indicates the significance level determined as explained in the text.
HJD - 2,400,000
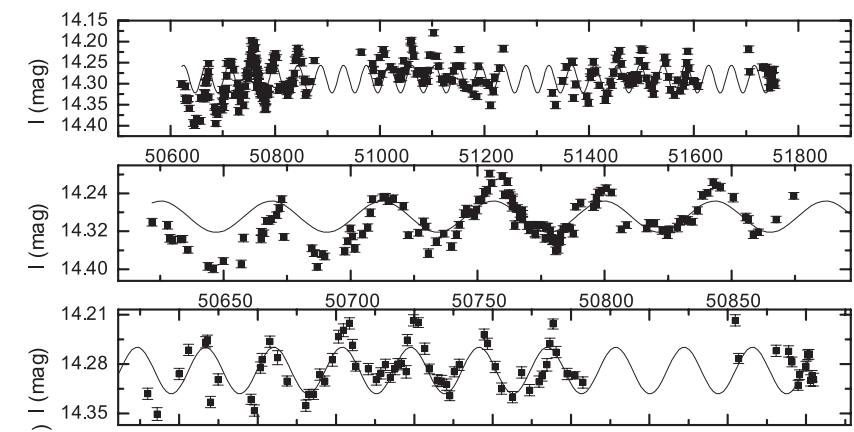

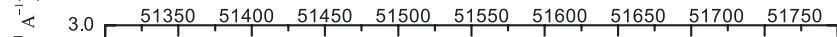

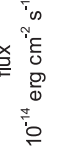

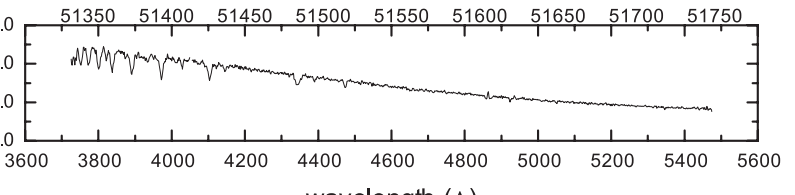

wavelength $(\mathrm{A})$
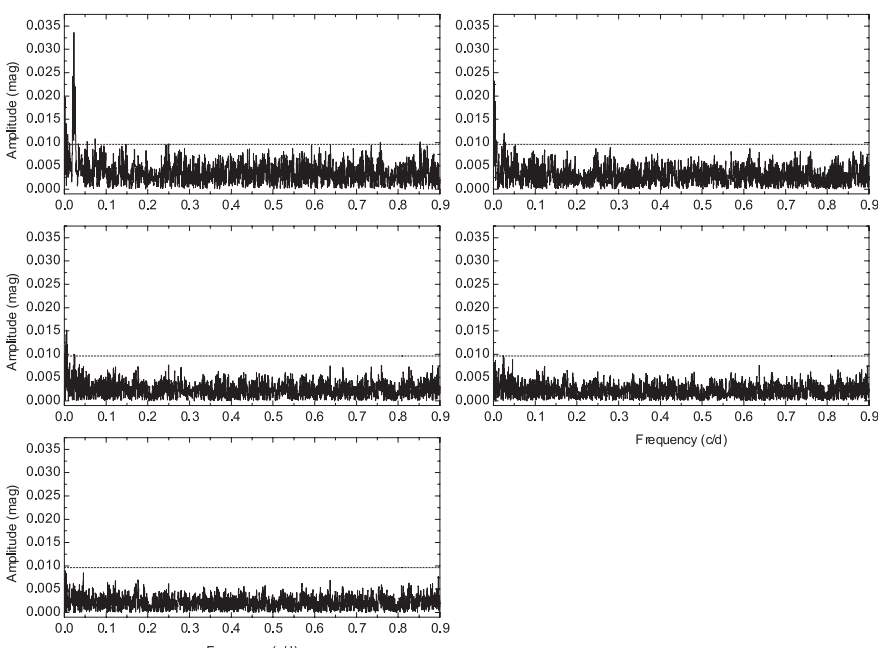

(1)

Fig. 17. a) OGLE light curve and the best-fitting function (upper), detail of the light curve and fit (middle) and spectrum (bottom) of SMC3-21. b) Fourier spectrograms illustrating the prewhitening process for SMC3-21. The dotted line indicates the significance level determined as explained in the text. 
R. E. Mennickent et al.: Long-period variables towards the SMC, Online Material $p 8$

Table 1. Journal of spectroscopic observations. The HJD zero point is 2452500.

\begin{tabular}{lccccc}
\hline \hline Object & ID & HJD (blue-spec) & exptime (s) & HJD (red-spec) & exptime (s) \\
\hline OGLE00433691-7326377 & SMC3-1 & 56.55708 & 900 & 58.55686 & 900 \\
OGLE00445466-7328029 & SMC3-2 & 56.49586 & 600 & $58.54036,58.54399,58.54763$ & 300 \\
OGLE00455414-7314043 & SMC3-3 & 56.50746 & 900 & $59.64039,59.64402,59.64766$ & 300 \\
OGLE00463376-7312043 & SMC3-4 & & & $59.50340,59.51515,59.52640$ & 900 \\
OGLE00475014-7313164 & SMC3-5 & 56.52092 & 900 & & \\
OGLE00492141-7258449 & SMC3-6 & 56.62733 & 900 & & 300 \\
OGLE00502564-7258071 & SMC3-7 & 56.53294 & 900 & & 900 \\
OGLE00504344-7327053 & SMC3-8 & 56.54504 & 900 & $58.52697,58.53061,58.53425$ & 500 \\
OGLE00510018-7253039 & SMC3-9 & 56.64137 & 900 & 58.59654 & 900 \\
OGLE00510759-7326366 & SMC3-10 & & & 59.54330 & \\
OGLE00535922-7235089 & SMC3-12 & 56.56884 & 900 & 59.65760 & \\
OGLE00552027-7237101 & SMC3-14 & 57.56598 & 900 & 58.60897 & \\
OGLE00574525-7235321 & SMC3-15 & 56.58069 & 900 & & 900 \\
OGLE00581258-7230485 & SMC3-16 & 56.65358 & 900 & 58.57019 & \\
OGLE01000078-7255229 & SMC3-18 & 56.59264 & 900 & & \\
OGLE01045121-7246469 & SMC3-20 & 56.60425 & 900 & & \\
OGLE01045299-7159188 & SMC3-21 & 56.61592 & 900 & & \\
\hline
\end{tabular}


R. E. Mennickent et al.: Long-period variables towards the SMC, Online Material $p 9$

Table 3. Results of the search for frequencies.

\begin{tabular}{|c|c|c|c|c|c|c|c|c|c|c|}
\hline OGLE-name & noise & label & freq. $(\mathrm{c} / \mathrm{d})$ & error & $A$ & error & phase & error & $\sigma$ & stable \\
\hline \multirow[t]{7}{*}{ smc3-1 } & 0.61 & F1 & 0.00844 & 0.00044 & 20 & 05 & 0.300 & 0.107 & 19 & $\mathrm{y}$ \\
\hline & & $\mathrm{F} 2$ & 0.06498 & 0.00002 & 18 & 01 & 0.477 & 0.010 & 14 & $\mathrm{y}$ \\
\hline & & F3 & 0.01261 & 0.00005 & 08 & 01 & 0.638 & 0.013 & 12 & $\mathrm{y}$ \\
\hline & & F4 & 0.02115 & 0.00007 & 07 & 01 & 0.029 & 0.022 & 12 & $\mathrm{y}$ \\
\hline & & F5 & 0.00416 & 0.00204 & 07 & 01 & 0.817 & 0.217 & 11 & $\mathrm{y}$ \\
\hline & & F6 & 0.02932 & 0.00975 & 05 & 04 & 0.078 & 0.288 & 10 & $\mathrm{y}$ \\
\hline & & F7 & 0.05439 & 0.00565 & 04 & 02 & 0.277 & 0.275 & 10 & $\mathrm{n}$ \\
\hline \multirow[t]{8}{*}{ smc3-2 } & 0.61 & F1 & 0.05733 & 0.00007 & 28 & 03 & 0.799 & 0.006 & 21 & $\mathrm{y}$ \\
\hline & & $\mathrm{F} 2$ & 0.06347 & 0.00001 & 27 & 01 & 0.780 & 0.003 & 10 & $\mathrm{y}$ \\
\hline & & F3 & 0.01013 & 0.00003 & 09 & 01 & 0.799 & 0.011 & 08 & $\mathrm{y}$ \\
\hline & & F4 & 0.06944 & 0.00009 & 04 & 01 & 0.913 & 0.028 & 08 & $\mathrm{y}$ \\
\hline & & F5 & 0.05367 & 0.01350 & 04 & 03 & 0.801 & 0.248 & 08 & $\mathrm{y}$ \\
\hline & & F6 & 0.88816 & 0.00356 & 03 & 01 & 0.876 & 0.216 & 07 & $\mathrm{n}$ \\
\hline & & F7 & 0.12666 & 0.00013 & 03 & 01 & 0.950 & 0.041 & 07 & $\mathrm{y}$ \\
\hline & & F8 & 0.55969 & 0.00433 & 03 & 01 & 0.793 & 0.118 & 06 & $\mathrm{n}$ \\
\hline \multirow[t]{3}{*}{ smc3-3 } & 0.37 & F1 & 0.00983 & 0.00005 & 04 & 00 & 0.229 & 0.017 & 05 & $\mathrm{y}$ \\
\hline & & F2 & 0.01146 & 0.00008 & 03 & 00 & 0.150 & 0.023 & 04 & $\mathrm{n}$ \\
\hline & & $\mathrm{F} 3$ & 0.34732 & 0.01610 & 02 & 00 & 0.825 & 0.150 & 04 & $\mathrm{n}$ \\
\hline \multirow[t]{2}{*}{ smc3-5 } & 0.67 & F1 & 0.03321 & 0.00003 & 08 & 01 & 0.612 & 0.012 & 07 & $\mathrm{n}$ \\
\hline & & F2 & 0.00135 & 0.00322 & 04 & 01 & 0.787 & 0.116 & 07 & $\mathrm{n}$ \\
\hline smc3-6 & 6.2 & F1 & 0.00509 & 0.00155 & 54 & 12 & 0.881 & 0.103 & 61 & $\mathrm{n}$ \\
\hline smc3-7 & 0.45 & F1 & 0.03661 & 0.00105 & 04 & 01 & 0.499 & 0.165 & 05 & $\mathrm{n}$ \\
\hline \multirow[t]{6}{*}{ smc3-8 } & 1.7 & F1 & 0.01933 & 0.00008 & 18 & 00 & 0.739 & 0.018 & 24 & $\mathrm{y}$ \\
\hline & & $\mathrm{F} 2$ & 0.02309 & 0.00857 & 11 & 01 & 0.475 & 0.156 & 23 & $\mathrm{y}$ \\
\hline & & F3 & 0.01170 & 0.00007 & 13 & 02 & 0.554 & 0.022 & 21 & $\mathrm{y}$ \\
\hline & & F4 & 0.02152 & 0.00012 & 12 & 01 & 0.963 & 0.078 & 20 & $\mathrm{y}$ \\
\hline & & F5 & 0.40727 & 0.00216 & 07 & 02 & 0.800 & 0.084 & 19 & $\mathrm{y}$ \\
\hline & & F6 & 0.03205 & 0.00014 & 07 & 01 & 0.804 & 0.043 & 18 & $\mathrm{y}$ \\
\hline \multirow[t]{3}{*}{ smc3-9 } & 1.6 & $\mathrm{~F} 1$ & 0.00199 & 0.00027 & 13 & 01 & 0.894 & 0.044 & 19 & $\mathrm{n}$ \\
\hline & & F2 & 0.01594 & 0.00288 & 15 & 00 & 0.882 & 0.203 & 17 & $\mathrm{y}$ \\
\hline & & F3 & 0.01743 & 0.00006 & 13 & 03 & 0.676 & 0.018 & 14 & $\mathrm{n}$ \\
\hline smc3-10 & 0.62 & F1 & 0.00541 & 0.00018 & 12 & 01 & 0.510 & 0.006 & 06 & $\mathrm{y}$ \\
\hline \multirow[t]{6}{*}{ smc3-12 } & 0.58 & F1 & 0.01378 & 0.00002 & 17 & 01 & 0.731 & 0.006 & 09 & $\mathrm{y}$ \\
\hline & & F2 & 0.00688 & 0.00006 & 06 & 01 & 0.986 & 0.021 & 07 & $\mathrm{y}$ \\
\hline & & F3 & 0.03007 & 0.00014 & 03 & 01 & 0.453 & 0.044 & 07 & $\mathrm{n}$ \\
\hline & & $\mathrm{F} 4$ & 0.01923 & 0.00038 & 03 & 01 & 0.075 & 0.076 & 07 & $\mathrm{n}$ \\
\hline & & F5 & 0.02762 & 0.00496 & 03 & 01 & 0.436 & 0.256 & 06 & $\mathrm{n}$ \\
\hline & & F6 & 0.00409 & 0.00012 & 03 & 01 & 0.474 & 0.034 & 06 & $\mathrm{n}$ \\
\hline \multirow[t]{6}{*}{ smc3-14 } & 1.7 & F1 & 0.00931 & 0.00004 & 23 & 02 & 0.404 & 0.011 & 23 & $\mathrm{y}$ \\
\hline & & $\mathrm{F} 2$ & 0.01875 & 0.00776 & 11 & 01 & 0.179 & 0.228 & 21 & $\mathrm{y}$ \\
\hline & & F3 & 0.03743 & 0.00012 & 09 & 01 & 0.658 & 0.033 & 20 & $\mathrm{y}$ \\
\hline & & F4 & 0.05608 & 0.03590 & 09 & 03 & 0.932 & 0.240 & 19 & $\mathrm{y}$ \\
\hline & & F5 & 0.02996 & 0.01100 & 08 & 01 & 0.933 & 0.251 & 18 & $\mathrm{n}$ \\
\hline & & F6 & 0.04657 & 0.00069 & 08 & 01 & 0.142 & 0.042 & 18 & $\mathrm{n}$ \\
\hline smc3-15 & 0.82 & F1 & 0.00412 & 0.00132 & 03 & 01 & 0.026 & 0.108 & 07 & $\mathrm{n}$ \\
\hline \multirow[t]{4}{*}{ smc3-16 } & 1.2 & F1 & 0.01672 & 0.00011 & 32 & 00 & 0.146 & 0.031 & 16 & $\mathrm{y}$ \\
\hline & & $\mathrm{F} 2$ & 0.03337 & 0.01210 & 11 & 04 & 0.800 & 0.222 & 14 & $\mathrm{y}$ \\
\hline & & F3 & 0.00588 & 0.00270 & 07 & 01 & 0.375 & 0.076 & 13 & $\mathrm{n}$ \\
\hline & & F4 & 0.01584 & 0.00514 & 06 & 01 & 0.495 & 0.075 & 12 & $\mathrm{n}$ \\
\hline \multirow[t]{5}{*}{ smc3-18 } & 2.2 & F1 & 0.02829 & 0.00141 & 24 & 06 & 0.853 & 0.166 & 27 & $\mathrm{y}$ \\
\hline & & $\mathrm{F} 2$ & 0.00086 & 0.00017 & 20 & 07 & 0.831 & 0.032 & 24 & $\mathrm{n}$ \\
\hline & & F3 & 0.02102 & 0.00012 & 12 & 01 & 0.027 & 0.050 & 23 & $\mathrm{n}$ \\
\hline & & F4 & 0.29259 & 0.00007 & 10 & 02 & 0.556 & 0.032 & 22 & $\mathrm{n}$ \\
\hline & & F5 & 0.01341 & 0.02090 & 11 & 01 & 0.030 & 0.162 & 21 & $\mathrm{n}$ \\
\hline smc3-20 & 1.1 & F1 & 0.02249 & 0.00006 & 09 & 01 & 0.119 & 0.018 & 10 & $\mathrm{y}$ \\
\hline \multirow[t]{4}{*}{ smc3-21 } & 2.4 & F1 & 0.02285 & 0.00429 & 34 & 00 & 0.008 & 0.374 & 33 & $\mathrm{y}$ \\
\hline & & $\mathrm{F} 2$ & 0.00143 & 0.00003 & 38 & 04 & 0.755 & 0.018 & 26 & $\mathrm{n}$ \\
\hline & & F3 & 0.00547 & 0.00011 & 16 & 03 & 0.408 & 0.025 & 24 & $\mathrm{n}$ \\
\hline & & F4 & 0.02347 & 0.01400 & 12 & 01 & 0.267 & 0.235 & 22 & $\mathrm{n}$ \\
\hline
\end{tabular}

